Published in final edited form as:

Lancet. 2019 January 12; 393(10167): 183-198. doi:10.1016/S0140-6736(18)32218-9.

\title{
Antibiotic allergy
}

\author{
Kimberly G Blumenthal ${ }^{1}$, Jonny G Peter ${ }^{2}$, Jason A Trubiano ${ }^{3}$, and Elizabeth J Phillips ${ }^{4}$ \\ 1.Division of Rheumatology, Allergy and Immunology, Department of Medicine, Massachusetts \\ General Hospital, Boston, MA, USA; Harvard Medical School, Boston, MA, USA. \\ 2.Division of Allergy and Clinical Immunology, Department of Medicine, University of Cape Town, \\ Cape Town, South Africa; Allergy and Immunology Unit, University of Cape Town Lung Institute, \\ Cape Town, South Africa. \\ 3.Department of Infectious Diseases, Austin Health, Melbourne, VIC, Australia; Department of \\ Medicine, University of Melbourne, Melbourne, VIC, Australia; The National Centre for Infections \\ in Cancer, Peter McCallum Cancer Centre, Melbourne, VIC, Australia. \\ 4.Institute for Immunology and Infectious Diseases, Murdoch University, Murdoch, WA, Australia; \\ Department of Medicine, Vanderbilt University Medical Center, Nashville, TN, USA.
}

\section{Abstract}

\begin{abstract}
Antibiotics are the commonest cause of life-threatening immune-mediated drug reactions that are considered off-target, including anaphylaxis, and organ-specific and severe cutaneous adverse reactions. However, many antibiotic reactions documented as allergies were unknown or not remembered by the patient, cutaneous reactions unrelated to drug hypersensitivity, drug-infection interactions, or drug intolerances. Although such reactions pose negligible risk to patients, they currently represent a global threat to public health. Antibiotic allergy labels result in displacement of first-line therapies for antibiotic prophylaxis and treatment. A penicillin allergy label, in particular, is associated with increased use of broad-spectrum and non- $\beta$-lactam antibiotics, which results in increased adverse events and antibiotic resistance. Most patients labelled as allergic to penicillins are not allergic when appropriately stratified for risk, tested, and re-challenged. Given
\end{abstract}

Corresponding author: Prof Elizabeth J Phillips, Department of Medicine, Vanderbilt University Medical Center, Nashville, TN 37232-2582, USA, elizabeth.j.phillips@ vanderbilt.edu.

Contributors

KGB performed the literature search and drafted the first report of this Review. All authors contributed equally to the development of visuals included in this Review and participated in the critical revision of the manuscript.

Declaration of interests

The authors declare no competing interests. KGB receives research funding and career development support from the National Institutes of Health, USA and the American Academy of Allergy, Asthma and Immunology Foundation, USA; has authored copywritten material and an electronic decision support tool and app used institutionally at Partners Health Care System for hospitalised patients with historical $\beta$-lactam allergies; has received royalties from Up To Date (Waltham, MA, USA) and honorarium from the New England Society of Allergy, USA. JGP is supported by a grant from the National Institutes of Health, USA (K43 TW011178-01) JAT is supported by a National Health and Medical Research Council (NHMRC) of Australia Early Careers Fellowship and a postdoctoral scholarship from the National Centre for Infections in Cancer (NCIC, Australia). EJP reports grants from NHMRC of Australia; grants from the National Institute of Health, USA (1P50GM11530501, 1P30AI110527-01A1, R21AI139021, and R34AI136815); grants from the Australian Centre for HIV and Hepatitis Virology Research (all paid to the institution); personal fees from Up To Date and Biocryst; consulting fees not directly received from Aicuris, outside the submitted work; and is co-director of IIID Pty Ltd that holds patent for HLA-B*57:01 testing for abacavir hypersensitivity, without any financial remuneration and not directly related to the submitted work. Funders played no role in any aspect of this Review. 
the public health importance of penicillin allergy, this Review provides a global update on antibiotic allergy epidemiology, classification, mechanisms, and management.

\section{Introduction}

Antibiotics can result in adverse drug reactions (ADRs) and hypersensitivity reactions (HSRs) through a variety of mechanisms. Antibiotic allergies are frequently documented in the electronic health record, which results in changes to the care of future infectious diseases. Inaccurately determined allergies might result in the use of unnecessarily broadspectrum or inferior antibiotics, posing a threat to patient safety and public health. Despite these threats, the histories associated with documented allergies are rarely reconciled, or acted on, by the health care team. Ideally, patients at low risk for allergy would have their allergy evaluated without specialist intervention, and high-risk patients would be referred for allergy diagnostic testing and have potential reaction mechanism(s) implicated. Although some allergy investigations are validated diagnostic tests approved by governing bodies globally, many tests for immunologically mediated drug hypersensitivity remain under investigation.

In this Review, we provide a global perspective on antibiotic allergies, with a focus on updated classification, epidemiology, effect on public health, diagnosis, and management. We also advise on the crucial steps required to appropriately combat unverified penicillin allergy labels as an emergent threat for individuals and public health.

\section{Classification, presentation, and mechanism}

ADRs include any untoward medication effect experienced at normal therapeutic doses of the drug, and HSRs are ADRs that are immunologically mediated. As our mechanistic understanding of ADRs improves, limitations of previous ADR classifications have become apparent. Consequently, a high-level classification of on-target and off-target reactions, with further categorisation of off-target immune and non-immune reactions has been proposed (figure 1). ${ }^{[2,3]}$ Both on-target and off-target effects can show concentration-exposure relationships that can differ between individuals, due to acquired or genetic host factors. The type and intensity of interaction between the drug and target may relate to both the dose and duration of treatment. This classification recognises that in many ADRs, for example druginduced liver injury, the aetiopathogenesis is not due to a singular mechanism. The targeting of eukaryotic and prokaryotic cellular differences by antibiotics means that there are few, true on-target antibiotic ADRs, which are due to an augmentation of the known therapeutic and pharmacological action of a drug. The negative consequences of disrupting commensal microbial communities, such as antibiotic-associated diarrhoea or Clostridioides difficile infection, are perhaps an exception. Some off-target ADRs are both directly immunemediated and associated with immunological memory of varied duration (drug hypersensitivity), whereas others without immunological memory might have an immunological phenotype, such as non-IgE-mediated mast-cell activation seen with the use of fluoroquinolones. In this scheme, immunologically mediated drug hypersensitivity comprises the antibody-mediated and T-cell-mediated off-target ADRs. 
Other HSR frameworks, including Gell and Coombs hypersensitivity mechanisms (eg, types I through IV HSRs) and reaction chronology and onset (immediate vs delayed), remain clinically important. Immediate antibiotic HSRs may either be mediated by IgE or by other factors (figure 1, table). The pathways of IgE-mediated reactions are well described. ${ }^{[6]}$ Recently a mechanism for some reactions not driven by IgE, previously called pseudoallergic or anaphylactoid, has been further elucidated. A receptor on murine mast cells, Mrgprb2, the orthologue of the human G-protein-coupled receptor MRGPRX2, was necessary for certain non-IgE-mediated drug reactions. ${ }^{[5]}$ Vancomycin and fluoroquinolones are the most commonly recognised mast-cell activators that cause non-IgE-mediated reactions to antibiotics, ${ }^{[8]},{ }^{[9]}$ thus producing a reaction with an immunological phenotype, but without immunological memory. Typically, non-IgE reactions have less cardiovascular symptomatology and hypotension, but are otherwise not easily distinguished from IgEmediated allergy (table). ${ }^{[10]}$

Delayed HSRs are mediated by T cells or antibodies other than IgE (table). Antibodymediated cytopenias, such as haemolytic anaemia, neutropenia, and thrombo-cytopenia (Gell and Coombs Type II), and serum sickness (Gell and Coombs Type III), are uncommon. Organ-specific HSRs to antibiotics often involve the liver (eg, drug-induced liver injury), kidney, or both (eg, acute interstitial nephritis). ${ }^{[3]}$ The commonest T-cell-mediated reaction to antibiotics is maculopapular rash, considered to be a type IVb HSR (table). ${ }^{[1]}$ This is the mechanism of the drug rash observed with aminopenicillin use. Other specific cutaneous HSRs from antibiotics include fixed drug eruptions, reported from tetracyclines, sulphonamides, $\beta$-lactams, vancomycin, and fluconazole. ${ }^{[2]}$ Generalised fixed drug eruptions can have bullae and mimic Stevens-Johnson syndrome or toxic epidermal necrolysis. ${ }^{[7]}$ Vancomycin is the most common antibiotic cause of linear IgA bullous disease, a blistering cutaneous adverse reaction that can also mimic Stevens-Johnson syndrome and toxic epidermal necrolysis. ${ }^{[12]}$ The major severe cutaneous adverse reaction (SCAR) phenotypes include Stevens-Johnson syndrome, toxic epidermal necrolysis, drug reaction eosinophilia and systemic symptoms (DRESS) syndrome, and acute generalised exanthematous pustulosis, which are detailed in the table. ${ }^{[1,2]}$

In addition to causing HSRs through immunologic mechanisms, drugs can also be implicated as the cause through coincidential association with a viral exanthem or through drug-infection interactions. ${ }^{[13]}$ A notable example of a drug-infection interaction is the rash observed with Epstein-Barr virus and amino-penicillin treatment, present in at least $30 \%$ of such patients. ${ }^{[14]}$ Bacterial (eg, rash and mucositis associated with Mycoplasma pneumoniae) and viral (eg, herpes simplex virus) infections are directly linked to the onset of erythema multiforme mimicking Stevens-Johnson syndrome. ${ }^{[13]}$ A more traditional illness that resembles Stevens-Johnson syndrome and toxic epidermal necrolysis has also been associated with viruses such as Coxsackie A6. ${ }^{[15]}$ Viral reactivation to human herpesvirus (HHV) 6 and 7, cytomegalovirus, and Epstein-Barr virus has been described and thought to occur as a consequence of regulatory T-cell expansion and the immune dysregulation associated with DRESS, rather than as a trigger of DRESS syndrome. ${ }^{[13]}$ 


\section{Epidemiology}

\section{Adverse drug reactions and hypersensitivity reactions}

ADRs account for more than $3 \%$ of hospital admissions ${ }^{[16]}$ and complicate the inpatient care of $10-20 \%$ of hospitalised patients. ${ }^{[17,18]}$ Drug HSRs comprise up to $20 \%$ of ADRs and are reported in approximately $8 \%$ of general populations. ${ }^{[19,20]}$ Cutaneous reactions, including rash and hives, are the most commonly reported HSRs. ${ }^{[21,22]}$ Although most patients are labelled with an antibiotic allergy at the time of hospital admission, new onset cutaneous HSRs were found to affect approximately $2 \%$ of inpatients. ${ }^{[11]}$ Severe, immediate allergies are infrequent; however, anaphylaxis comprised $3 \%$ of reactions documented in a US electronic health record repository of allergy. ${ }^{[21]}$

Early studies identified antibiotics, particularly $\beta$-lactams, as the most common HSR culprits. ${ }^{[11]}$ However, antibiotic HSRs are easily misdiagnosed because alternative explanations for rashes exist (eg, infections from viruses such as Herpesviridae, or bacteria such as Streptococcus pyogenes, and drug-infection interactions). ${ }^{[14,22],[23]}$ Antibiotic allergy labels, which are those documented in health records but unverified, might also be recorded incorrectly in patients' charts after a non-immunological reaction, such as gastrointestinal upset, headache, or fatigue. ${ }^{[21]}$

$\beta$-Lactams, which include penicillins, cephalosporins, carbapenems, and monobactams (figure 2), are the most common antibiotic classes reported to cause HSRs. ${ }^{[24,25]} \beta$-Lactam ADRs are documented in $5-15 \%$ of patients' charts. ${ }^{[25,26]}$ Sulphonamide antibiotics are another commonly reported antibiotic allergy, with ADRs documented in 2-10\% of cases. [24-26] Patients labelled as sulfa allergic could have had a reaction previously to sulphonamide antibiotics or a non-antibiotic sulphonamide, and notably there is no crossreactivity between sulphonamide antibiotics and non-antibiotic sulphonamides. ${ }^{[27]}$ Sulphonamide antibiotics are implicated in benign T-cell-mediated rashes and SCARs. ${ }^{[1,28]}$ A third of reported cases of Stevens-Johnson syndrome and toxic epidermal necrolysis documented in electronic health records is attributed to sulphonamide antibiotics. ${ }^{[25,29]}$

Other notable antibiotic allergies reported to cause HSRs are fluoroquinolones, macrolides, tetracyclines, and glycopeptides. ${ }^{[25,30]}$ Although these antibiotic classes generally cause cutaneous reactions, the glycopeptide vancomycin is also the commonest antibiotic implicated in non-IgE-mediated reactions and up to $40 \%$ of DRESS syndrome cases. [12,29,31-34]

\section{$\beta$-Lactam antibiotics}

Penicillin was first widely used in the 1940s, with reports of immediate drug hypersensitivity surfacing soon thereafter. ${ }^{[34]}$ Early reported allergies to penicillins included injection reactions, serum sickness-like reactions, and delayed T-cell-mediated cutaneous eruptions. Studies confirm an approximate penicillin reaction rate from $0.5 \%$ to $5.0 \%$ of administrations. ${ }^{[8,11]}$ Today, from $5 \%$ to $15 \%$ of patients in developed countries carry a penicillin allergy label. ${ }^{[24-26,35]}$ Aminopenicillins, largely administered orally, have been used since the 1970s. Although they are recognised as the most common cause of drug- 
induced delayed rashes and drug viral interactions, ${ }^{[14]}$ they infrequently cause true IgEmediated reactions.

In the USA, cephalosporin ADRs are documented in 1-2\% of patients' charts, ${ }^{[36]}$ with rash being the most commonly reported reaction. The use of carbapenems is uncommon globally and is often restricted by antimicrobial stewardship programmes, because of the drugs' broad-spectrum activity and formulation as parenteral and intramuscular antibiotics. As such, ADRs and HSRs reported from carbapenems are substantially lower than those reported from penicillins and cephalosporins. ${ }^{[24,25]}$

\section{$\beta$-Lactam IgE-mediated HSRs}

Although IgE-mediated reactions are not uncommon in patients treated with penicillin, anaphylaxis is rare (approximately $0.001 \%$ for parenteral exposures and $0.0005 \%$ for oral exposures). ${ }^{[37,38]}$ IgE-mediated penicillin HSRs are less frequent today than described previously, and the prevalence of penicillin anaphylaxis has also declined over time. ${ }^{[39]}$ There was one fatal amoxicillin reaction in the UK during the period from 1972 to 2007. [37] The changing epidemiology of IgE-mediated penicillin allergy might be attributed to newer, less allergenic formulations and changes in administration route. ${ }^{[40,41]}$ Penicillin antibiotics commonly prescribed today are used orally, such as for bacterial pharyngitis, sinusitis, lower respiratory tract infections, or skin and soft tissue infections. In addition to oral administration, cephalosporins are vital intramuscular (eg, ceftriaxone) and parenteral (eg, cefazolin, cefepime, ceftriaxone) anti- biotics. The cephalosporin cefazolin is identified as a common causative agent in perioperative anaphylaxis in countries where it is available and frequently used (USA, Canada, UK, France, Australia, South Africa, and parts of Southeast Asia and South America). ${ }^{[8]}$

\section{Other $\beta$-lactam HSRs}

The most common $\beta$-lactam reaction is a delayed-type rash, often a T-cell-mediated eruption. $\beta$-Lactams are also key culprits in serum sickness-like reactions observed that are due to cephalosporins, often cefaclor, and penicillins, typically with high-dose parenteral penicillin therapy. ${ }^{[8]}$

SCARs are the most severe non-immediate HSRs and can be attributed to antibiotics in a quarter to half of cases. ${ }^{[33,42]}$ A recent USA-based study calculated an annual incidence per million inhabitants of 8.61-9.69 cases for Stevens-Johnson syndrome, 1.46-1.84 cases for Stevens-Johnson syndrome and toxic epidermal necrolysis overlap, and 1.58-2.26 cases for toxic epidermal necrolysis. ${ }^{[43]}$ Antibiotics, including penicillins, are reported as SCAR culprits but are also common drugs started at the first sign of the Stevens-Johnson syndrome and toxic epidermal necrolysis prodrome that mimics an infection. ${ }^{[32]}$ Illnesses similar to Stevens-Johnson syndrome and not induced by drugs, such as erythema multiforme, are often mis-classified as Stevens-Johnson syndrome, and the anti-biotics and non-steroidal anti-inflammatory drugs introduced during the prodromal stage of illness may be implicated as causative. ${ }^{[7]}$ Patients with antibiotic-associated SCAR are often treated with more than one antimicrobial at the time of diagnosis. Aminopenicillins and cephalosporins uncommonly cause SCARs but may be implicated when drug causality is unclear. ${ }^{[28]}$ In a 
US study of over 800000 patients exposed to over 1 million cephalosporins, there were three cases of cephalosporin-associated SCARs documented, but patients were on other drugs that could also have caused the SCAR. ${ }^{[44]}$

\section{Special patient groups}

The frequency of documented drug allergy is higher in women, those of self-reported European ancestry, adults, and in inpatients. ${ }^{[20,24]}$ Female predominance has been stable across multiple studies for reported allergies, especially for antibiotic allergies, but no sex effect has been demonstrated in children. ${ }^{[24,25,45]}$ Patients whose self-determined ancestry is European report more IgE-mediated HSRs. ${ }^{[39]}$ Genetic associations for SCAR risk to specific drugs are more relevant in certain populations in which allele frequencies are higher, for example self-reporting Han Chinese or Black African (table). ${ }^{[1]}$ Adults have more selfreported drug allergy because of more cumulative drug exposures (ie, the strongest drug allergy risk factor). Almost a quarter of patients admitted to hospital have an antibiotic ADR documented in the allergy section of their electronic health records. ${ }^{[46]}$ Both penicillin and cephalosporin allergy labels are more common among inpatients and those linked to ongoing ambulatory care, compared with single-visit outpatients. ${ }^{[24,44]}$ Internationally, a penicillin allergy label among patients admitted to hospital ranges from 6\% (Netherlands) to $19 \%$ (Canada), although data from low-income and middle-income countries are scarce. ${ }^{[35,46-48]}$

Patients with documented allergies to multiple unrelated drugs or antibiotics are considered to have multiple drug allergy syndrome, which affects $1-5 \%$ of patients seeking health care. ${ }^{[22,49]}$ Such patients might have more depression, anxiety, and somatic illnesses, but this syndrome could have a biological basis in differential histamine-releasing factors, tolerances of small chemicals, drug-induced interferon gamma release, or pre-activated CD4 T cells. ${ }^{\text {[49] }}$ Patients with multiple drug allergy syndrome have allergy labels that interfere with optimal medical care and they often have subjective symptoms when drug allergies are formally evaluated. ${ }^{[24,49]}$

A high prevalence (23-35\%) of reported antibiotic allergy is observed in patients with cancer. ${ }^{[50,51]}$ Patients with HIV/AIDS also have a high frequency of reported drug allergy (up to one in four); these patients have 10-100 times more cutaneous reactions caused by drugs (including SCARs) than individuals without HIV/AIDS, especially from sulphonamide antibiotics. ${ }^{[52,53]}$ Over $10 \%$ of patients with HIV have a reported sulphonamide antibiotic allergy or intolerance, ${ }^{[54]}$ although data from endemic populations are insufficient. Compared with patients without cystic fibrosis, patients with cystic fibrosis have a threefold higher incidence of antibiotic allergy, with approximately a third of patients reporting an antibiotic allergy. ${ }^{[55]}$ Although this high frequency might be related to high drug-infection interactions or a need for high-dose parenteral antibiotic treatment, most reactions in patients with cystic fibrosis are not IgE-mediated. ${ }^{[56]}$

\section{Unverified antibiotic allergy labels}

Most patients labelled with a $\beta$-lactam allergy are not allergic (ie, they tolerate penicillin and related drugs). ${ }^{[57]}$ This mislabel occurs for a variety of reasons. First, the original reaction might not have been an allergy (there could be intolerance, a viral exanthem, or a drug- 
infection interaction). Even if the original reaction were immunological, it might not recur with re-challenge. IgE-mediated reactions to $\beta$-lactams can wane over time; approximately $80 \%$ of patients who are positive for a penicillin skin test and $60 \%$ of those positive for a cephalosporin skin test are no longer sensitive, as measured by skin testing after a period of 10 and 5 years, respectively. ${ }^{[58,59]}$ Mild delayed reactions that in many cases were T-cellmediated do not reliably occur with re-challenge; ${ }^{[60-62]}$ such reactions, therefore, either did not represent adaptive immune responses or were immune responses that were lost in the absence of ongoing drug exposure.

Among patients admitted to hospital with a documented penicillin allergy who were skin tested and challenged, 95\% were not allergic and were de-labelled. ${ }^{[63]}$ Outpatients with documented penicillin allergies have also been largely (>98\%) tolerant to penicillin. ${ }^{[64,65]}$ However, notable global variation in the frequency of confirmed IgE-mediated penicillin allergy exists. Although some international variation might be tied to differential antibiotic prescribing patterns, other variations could be explained by differences in patient selection or demographic and genetic differences. For example, European studies confirm penicillin allergy in $18 \%-30 \%$ of evaluated patients, although confirmed allergy could include diagnostics in vitro. ${ }^{[66,67]}$

Children might have an even lower incidence of true $\beta$-lactam allergy because the observed allergy could have been confused with a viral exanthem. Most children with documented $\beta$ lactam allergies presenting to a US emergency department (76\%) were determined to have low-risk allergy histories, unlikely to represent true allergy. ${ }^{[68,69]}$ Protocols in children have recently included one-step amoxicillin challenge without preceding skin testing and more than $90 \%$ had no immediate reactions. ${ }^{[61,70]}$

Although validated skin tests do not exist for non-penicillin antibiotics, skin testing with non-irritating concentrations and challenge procedures have identified that $11 \%$ of US patients in one study ${ }^{[71]}$ and less than $1 \%$ in another ${ }^{[72]}$ were allergic to the drug reported to cause an allergy that prompted specialist evaluation. In European studies, less than $20 \%$ of patients with reported reactions have their allergy confirmed. ${ }^{[73]}$ Therefore, more than $80 \%$ of patients seen by allergy specialists for evaluation of non-penicillin antibiotic allergies are likely tolerant. Although such patients could also benefit from drug allergy evaluations to confirm them or rule them out, to date, there are no direct data supportive of the need for such evaluations for improved quality, safety, and public health.

\section{Effect of antibiotic allergy labels}

Precise assessment and subsequent documentation of antibiotic allergies is a key mechanism to ensure patients do not receive a medication to which they are allergic. However, most allergy labels are untrue and less than $1 \%$ of reported antibiotic allergies globally are interrogated through allergy evaluation methods, despite known negative consequences of allergy mislabels for patients, health-care systems, and communities. 


\section{Effect on patients}

Patients with only a penicillin allergy documented receive alternative antibiotics that are more broad-spectrum and have lower efficacy or increased side effects, such as vancomycin, clindamycin, gentamicin, and fluoro- quinolones. ${ }^{[47]}$ Alternatives are used even when $\beta$ lactams are indicated. ${ }^{[74,75]}$ Canadian inpatients with a $\beta$-lactam allergy label had a threefold increased risk of adverse events, compared with patients without a documented $\beta$ lactam allergy. ${ }^{[48]}$

\section{Effect on health-care associated infections}

Antibiotic allergies have a strong impact on the development of health-care associated infections, which are globally and uniformly important to patients, hospitals, and health-care systems. These infections are monitored for quality, safety, and public health purposes. ${ }^{[76]}$

The US Centers for Disease Control and Prevention consider C difficile infections an urgent threat to public health with over half a million cases annually. ${ }^{[77]}$ Prevalence of this infection type was increased by $23 \%$ in US patients admitted to hospital with penicillin allergy labels compared with those without a penicillin allergy label. ${ }^{[78]}$ Patients with penicillin allergy in a UK cohort had a $26 \%$ increased incidence of $\mathrm{C}$ difficile infection, compared to matched comparators after adjustment for other known $\mathrm{C}$ difficile risk factors. ${ }^{[9]}$ Over a third of the heightened $\mathrm{C}$ difficile risk in patients with penicillin allergy was attributable to subsequent $\beta$-lactam alternative antibiotic use, with subsequent fluoroquinolone use alone responsible for more than $10 \%$ of the increased risk. ${ }^{[79]}$

Infections that occur postoperatively, termed surgical site infections, represent almost half of health-care associated infections ${ }^{[80]}$ and result in substantial patient morbidity. ${ }^{[81]}$ When patients with penicillin allergy labels get surgical site infections, inferior perioperative prophylactic antibiotic choice may be the cause. ${ }^{[82]}$ For most surgical procedures, the $\beta$ lactams cefazolin or cefoxitin are the preferred perioperative antibiotics. ${ }^{[83]}$ For patients who report a previous penicillin allergy, the non- $\beta$-lactam antibiotics clindamycin, vancomycin, or teicoplanin are often administered, even though there is very limited and unproven crossreactivity between penicillins and cefazolin in patients with a documented IgE-mediated allergy to penicillin (figure 2). ${ }^{[84]}$ Among 8385 perioperative patients in the USA, penicillin allergy labels resulted in $50 \%$ increased odds of surgical site infections attributed to perioperative antibiotic choice or timing, compared with patients without a penicillin allergy label. ${ }^{[82]}$ Alternative non- $\beta$-lactam antibiotics such as clindamycin and vancomycin can also confer additional negative sequelae, including postoperative $\mathrm{C}$ difficile infections ${ }^{[85]}$ and non-IgE-mediated reactions respectively, even when used sparingly in the perioperative setting. ${ }^{[86,87]}$

\section{Effect on antibiotic resistance}

Each year in the USA, at least 2 million people become infected with bacteria that are resistant to antibiotics, with at least 50000 Americans and Europeans dying annually as a direct result of these infections. ${ }^{[77,88]}$ A UK report predicted that 10 million people globally could die from antimicrobial resistance per year by $2050 .{ }^{[88]}$ Some of the most common resistant pathogens include methicillin-resistant Staphylococcus aureus (MRSA) and 
vancomycin-resistant Enterococci (VRE). One previous study documented a 14\% increased prevalence of MRSA and 30\% increased prevalence of VRE in hospital inpatients with a penicillin allergy matched to those without a penicillin allergy label. ${ }^{[78]}$ A UK study identified that a penicillin allergy label conferred a $69 \%$ increased incidence of MRSA ${ }^{[79]}$ and $55 \%$ of the increased risk was attributable to administration of $\beta$-lactam alternative antibiotics.

One of the core actions recommended to prevent antibiotic resistance is improving antibiotic prescribing and stewardship, ${ }^{[89]}$ which includes penicillin allergy evaluations as a method to reclaim narrow-spectrum $\beta$-lactams. ${ }^{[90]}$ International guidelines have begun to recommend penicillin allergy assessments as part of antibiotic stewardship interventions.91

\section{Diagnosis and management of suspected hypersensitivity}

The evaluation of patients with antibiotic allergies begins with an allergy history that includes symptom details, timing of reaction, timing since reaction, treatment of the reaction, and relevant ingestions concurrent with, and since, the reaction. When relevant, review of historical details, such as: rash description, photos, and biopsy; concomitant medication list; concomitant diagnoses; laboratory; and imaging details should be obtained. Although allergy specialists widely agree on these important history components, limited drug allergy history tools have been developed, endorsed, and validated. ${ }^{[92]}$ Further, tools have largely been for specialist use, although a practical history risk tool that uses low-risk and high-risk signals from the salient history is needed (figure 3). Drug allergy history tools for general use have included clinical decision support for inpatient providers ${ }^{[3,94]}$ and a history tool for perioperative patients implemented by pharmacists. ${ }^{[95]}$

\section{Potentially IgE-mediated reactions}

Patients with reactions that are, by history, immediate and potentially IgE-mediated can undergo further evaluation (figure 4). Although it is appropriate for this initial evaluation and risk-stratification to be performed by non-specialists, patients with severe immediate or delayed reactions should be evaluated by the relevant specialist, such as an allergist or dermatologist.

For reactions that could be IgE-mediated, skin testing can be considered (figure 4, appendix). See online for appendix). Antibiotic skin testing for immediate reactions uses both epicutaneous (ie, prick, puncture, or scratch) testing and intradermal skin testing (if the epicutaneous step is negative). For penicillin skin testing, the major antigenic determinant penicilloylpolylysine (also known as PPL) injection is used ${ }^{[8,96,99]}$ and is available as the PRE-PEN ${ }^{[97]}$ or Diater DAP-kit. ${ }^{[98]}$ Skin tests for drugs and drug antigens are performed and compared with a positive control (histamine phosphate) and a negative control (normal saline). Penicillin skin testing has been successfully implemented by internists, ${ }^{[100]}$ infectious diseases physicians, ${ }^{[101]}$ and pharmacists, ${ }^{[102]}$ largely in patients with non-severe allergy phenotypes.

To skin-test patients for immediate reactions to anti-biotics other than penicillin, nonirritating concentrations are used. ${ }^{[103]}$ Antibiotics that typically cause non-IgE- mediated 
reactions, such as fluoroquinolones and vancomycin, have measurable non-specific mast-cell activation that renders immediate hypersensitivity skin testing challenging to interpret. ${ }^{[8]}$

Drug challenge procedures, whereby a therapeutic dose of the culprit drug is administered under medical observation, are the current standard for excluding IgE-mediated allergy. Challenge procedures are often performed using escalating drug doses in one, two, or three steps, and 30-60 min of observation in between steps. A common challenge to disprove IgEmediated penicillin allergy is a two-step amoxicillin challenge, for example administering 50 $\mathrm{mg}$ of amoxicillin orally with an observation period of 30-60 min. If there is no reaction, then $500 \mathrm{mg}$ is administered orally, followed by another period of observation of 60-90 min. A common one-step amoxicillin challenge for patients at low risk of allergy is simply the administration of 250-500 $\mathrm{mg}$ of amoxicillin to a patient and observing them for 60-120 min. In patients at high risk for IgE-mediated allergy, skin testing should precede drug challenge, when available. The skin test and challenge together have more than $99 \%$ negative predictive value for excluding IgE-mediated penicillin allergy. ${ }^{[8]}$ Drug challenge procedures for patients labelled with penicillin allergy have been implemented in pediatric outpatients, ${ }^{[61,104]}$ military recruits, ${ }^{[65]}$ hospitalised patients, ${ }^{[93]}$ and allergy outpatients. $[60,71,72]$

Cross-reactivity between $\beta$-lactam antibiotics has been described for IgE-mediated HSRs (figure 2). ${ }^{[84]}$ Early cephalosporin formulations were likely to be contaminated with penicillin, leading to high estimates of $\beta$-lactam cross-reactivity (10\%). ${ }^{[105]}$ Although the cross-reactivity rate is currently calculated to be lower than these initial estimates $(2 \%),{ }^{[8]}$ European allergy referral populations have documented high rates of $\beta$-lactam crossreactivity in skin tests, predicted by shared side-chain structures. ${ }^{[106,107]}$

Testing can often be able to distinguish a non-IgE-mediated reaction from an IgE-mediated reaction. If a serum mast-cell tryptase was drawn at the time of a reaction and elevated, an $\operatorname{IgE}$ (rather than non-IgE) mechanism is likely. ${ }^{[8]}$ For clear non-IgE-mediated mast-cell activation, future administrations require pre-medications, slowed infusions, or altering drug choice (table). When an IgE mechanism is excluded, future antibiotic use is considered safe; however, few longer-term studies exist. We know that patients with previous penicillin allergy who had negative penicillin allergy evaluation received a subsequent series of parenteral courses of penicillin without difficulty. ${ }^{[108]}$ However, despite a negative IgE allergy evaluation, approximately $3 \%$ of adult patients and up to $10 \%$ in pediatric patients ${ }^{[61]}$ could have a benign, delayed, possibly T-cell-mediated eruption to the drug. ${ }^{[109]}$ These reactions are nevertheless considered to be close to their baseline incidence in the general population. ${ }^{[11]}$ Although some allergists advocate for prolonged multiple-day oral challenges of 3,5 , or 7 days to ensure there is no evidence of delayed hypersensitivity, ${ }^{[110]}$ general antibiotic stewardship principles caution against unnecessary antibiotic usage. Therefore, prolonged multiple drug challenges need to only be employed in carefully selected patients. ${ }^{[62]}$

When IgE-mediated allergy is confirmed by skin testing or drug challenge, patients can only receive the drug in question by an induction of tolerance or desensitisation procedure (appendix) ${ }^{[111]}$ For patients whose clinical history alone is high-risk for true, IgE-mediated 
allergy (eg, severe or recurrent immediate reactions), or in situations in which anaphylaxis would pose an unacceptable risk (eg, those with unstable coronary or respiratory status or pregnancy), desensitisation procedures can be used without skin testing to safely administer a first-line antibiotic therapy despite the allergy. ${ }^{[112]}$ Desensitisations are particularly beneficial to facilitate use of $\beta$-lactam antibiotics when alternatives have inferior efficacy (eg, methicillin-sensitive Staphylococcus aureus endocarditis or bacteraemia, streptococcal or enterococcal endocarditis and syphilis in pregnancy). ${ }^{[8,74]}$

\section{Non-immediate reactions}

For non-immediate reactions, delayed intradermal testing or patch testing can be used (figure 4). Delayed intradermal testing is more convenient for patients than patch testing, as multiple reads are not required and positives can be identified within $24 \mathrm{~h}$. It also appears more sensitive than patch testing for DRESS and acute generalised exanthematous pustulosis. For Stevens-Johnson syndrome and toxic epidermal necrolysis, in which delayed intra-dermal testing is contraindicated despite a low risk of provoking a systemic reaction, the sensitivity of patch testing is less than $30 \%$ and is therefore not recommended unless the benefit outweighs any risk. Patch testing is generally avoided when the culprit drug can be identified with high likelihood on the basis of clinical history alone. ${ }^{[113-115]}$ Patch testing is performed by applying a drug in a soluble base (usually petroleum), with subsequent patch removal after $48 \mathrm{~h}$ and taking readings for erythema, induration, and vesiculopapular eruption at $48 \mathrm{~h}, 96 \mathrm{~h}$, and 7 days to maximise sensitivity. Patch testing has proved clinically useful for specific drug hypersensitivity phenotypes (eg, acute generalised exanthematous pustulosis, intra-lesional fixed drug eruptions) and culprit drugs (eg, abacavir hypersensitivity syndrome). ${ }^{[116,117]}$

For non-SCAR T-cell-mediated hypersensitivity, re-challenge is safe and cross-reactivity is less defined. ${ }^{[8]}$ Administration of small, escalating doses over hours, days, and weeks have also been successfully used in patients with reported non-SCAR T-cell-mediated hypersensitivity, typically for delayed rashes from a sulphonamide antibiotic. ${ }^{[112,118]}$

For severe T-cell-mediated reactions, such as Stevens-Johnson syndrome and toxic epidermal necrolysis, DRESS, and organ-specific reactions, there are few long- term antibiotic re-challenge or cross-reactivity data to guide future therapy. ${ }^{[32]}$ However, since exvivo and in-vitro studies have demonstrated long-lived immune responses, ${ }^{[119]}$ patients with severe T-cell-mediated allergies associated with antibiotics should refrain from re-exposure to the same drug and, ideally, all potentially cross-reactive drugs. The exception to this is when SCAR occurs in the setting of multiple drug therapy for tuberculosis, in which the benefit of selective drug re-challenges might outweigh the risk of death from an inadequately treated infection. ${ }^{[53]}$ The SCAR should remain a permanent part of the patients' allergy history. ${ }^{[3]}$

\section{New and investigational allergy tools}

Advancing diagnostic testing for drug HSRs requires distinguishing patients who are reportedly allergic from those who are truly allergic with subsequent phenotyping and translational studies. To date, this research has been hampered by the disproportionate 
labelling of allergy, lack of standard time from HSR to clinical presentation to allergy specialists, and lack of known antigens for most drug allergens. Despite this, new investigational tools are being evaluated for both immediate and non-immediate HSRs (appendix, figure 4).

\section{A global call for action}

Although penicillin allergy evaluations are recognised as important by a variety of government bodies, foundations, and professional organisations, ${ }^{[91,120-122]}$ there is no standard approach to penicillin allergy evaluation or documentation. However, a systematic approach to remove the penicillin allergy label is now warranted.

Global implementation of penicillin allergy evaluations must be supported on an international scale to improve the quality and safety of health care delivered to patients with documented penicillin allergies. The simplest intervention might be a universal drug allergy history tool aimed at improving allergy documentation and identifying patients with penicillin allergy histories that should undergo further investigation. Even when there are limited allergy details, most patients will describe low-risk history elements (figure 3). Patients at low risk are most appropriate for de-labelling with direct re-challenge procedures. For patients at moderate risk for IgE-mediated allergy by history, de-labelling can be accomplished by first using penicillin skin testing, followed by a drug challenge for those with negative skin test results. Although penicillin skin testing was developed in the 1960s, and the primary reagent penicilloylpolylysine is commercially available, no clear guidance for its use exists on a global scale. Non-allergists need instruction and training on how to perform and interpret skin tests.

Given the large numbers of patients with documented penicillin allergy, evaluation programmes must prioritise immunocompromised, preoperative, or actively infected patients first and use different methods to remove the penicillin allergy label in low-risk patients, such as history alone, direct re-challenge, and skin testing. Variation by treatment setting must also be encouraged, since there are limitations in the inpatient setting that could make skin testing less desirable than drug challenges, ${ }^{[93,123]}$ whereas in preoperative settings skin testing might be preferable to direct challenges. ${ }^{[124-126]}$ There are existing treatment algorithms, questionnaires, and electronic clinical decision support systems for patients with $\beta$-lactam allergies, some of which consider direct cephalosporin use in patients reporting penicillin allergy (figure 5). ${ }^{[61,94,123,127,128]}$ Similar treatment algorithms have increased first-line antibiotic therapy and increased use of $\beta$-lactam antibiotics overall. ${ }^{[57,74,127]}$

Although allergists have unique expertise that make them suited to evaluate patients with suspected drug allergy, there is an inadequate supply of allergy specialists to address this problem alone. ${ }^{[129,130]}$ A quarter of US infectious diseases specialists describe not having any local options for antibiotic allergy testing, ${ }^{[131]}$ with similar deficiencies noted in Australia and New Zealand. ${ }^{[132]}$ In the UK, wait time to see an allergist exceeds 3 months. ${ }^{[87]}$ When straightforward, investigations for low-risk penicillin allergy can be accomplished by generalists throughout the world, then the complex cases can be appropriately triaged to allergists and specialist centres. 
There are many examples of penicillin allergy evaluations led by trained non-allergists with various specialist medical backgrounds. ${ }^{[95,100,101,133,134]}$ The most impactful multidisciplinary antibiotic allergy testing programmes have been those embedded in antimicrobial stewardship services. ${ }^{[57,102,127,128]}$ However, barriers to engaging nonallergists in penicillin allergy evaluations remain, as drug allergy is not universally taught in medical school, and Allergy and Immunology rotations for postgraduate trainees are not required in most centres outside of Europe. ${ }^{[135]}$ Most survey studies of general provider knowledge identified substantial educational gaps in the knowledge of drug allergy. [136,137] Thus, any intervention must include appropriate, multi-dimensional education for healthcare team members on the importance of penicillin allergy verification, drug allergy history taking, testing indication and methods, electronic health record documentation, and the implications of negative testing.

The education of patients and the general public is similarly crucial to advance penicillin allergy evaluations. Patients often use the term allergy interchangeably with side-effects, and most electronic health records contain missing, erroneous reactions that are inconsistently and incompletely documented ${ }^{[138,139]}$ or entirely discrepant with the patient report. ${ }^{[140]}$ Educating and empowering patients to define and manage their drug allergies and intolerances might improve allergy documentation generally and lead to more penicillin allergy evaluations and de-labelling. Patient education must also focus on the harms of unverified penicillin allergies, since patients can be resistant to allergy testing. Previous studies have shown that more than $15 \%$ of patients decline penicillin evaluation with skin testing when offered. ${ }^{[127,141]}$ Multimedia educational materials might also serve to assuage fear of future reactions in those found not allergic. For example, 18\% of parents refused penicillins for their child because of continued fear of a penicillin reaction, despite a negative penicillin allergy evaluation. ${ }^{[142]}$ Patient education has the potential to affect the uptake and effectiveness of any penicillin allergy evaluation programme.

\section{Conclusions}

Although antibiotic ADRs are commonly reported, immunologically mediated hypersensitivity is uncommon and true IgE-mediated antibiotic allergy is verified in only a small minority. For those with true antibiotic HSRs, appropriate specialty assessment is indicated to prevent future ADR-related morbidity and mortality. This assessment includes defining the most likely drug implicated in the allergic reaction, the probable mechanism(s), and the potential cross-reactive drugs that should be avoided in the future. Despite the threat associated with true antibiotic allergy, the highest burden lies with those reporting a penicillin allergy who do not have one. These patients have multiple lifelong negative sequelae that begin with inferior and unnecessarily broad-spectrum infection prophylaxis and treatment. Given the associations between unverified penicillin allergy and health-care associated infections and multidrug-resistant organisms, the capacity to appropriately address penicillin and other antibiotic allergy labels must increase globally across healthcare settings. To address this threat, international efforts might better define risk groups, determine optimal use and method of penicillin allergy evaluations, and identify a workforce to spread evaluations to environments in which the epidemiology of antibiotic allergy differs. Along with a strategic implementation plan, health-care provider and patient 
education materials and support are required. Despite the anticipated barriers to penicillin allergy de-labeling programmes, the benefits of reclaiming $\beta$-lactams are to improve infectious disease care and antibiotic stewardship, while unmasking drug hypersensitivity phenotypes to advance drug hypersensitivity discovery, outcomes that are desirable, feasible, and imminently necessary.

\section{Supplementary Material}

Refer to Web version on PubMed Central for supplementary material.

\section{Acknowledgments}

We thank Yu Li for her research assistance and Karen Adamson for her design expertise.

\section{References}

1. Duong TA, Valeyrie-Allanore L, Wolkenstein P, Chosidow O. Severe cutaneous adverse reactions to drugs. Lancet 2017; 390: 1996-2011. [PubMed: 28476287]

2. Peter JG, Lehloenya R, Dlamini S, et al. Severe delayed cutaneous and systemic reactions to drugs: a global perspective on the science and art of current practice. J Allergy Clin Immunol Pract 2017; 5: 547-63. [PubMed: 28483310]

3. White KD, Chung WH, Hung SI, Mallal S, Phillips EJ. Evolving models of the immunopathogenesis of T cell-mediated drug allergy: the role of host, pathogens, and drug response. J Allergy Clin Immunol 2015; 136: 219-34. [PubMed: 26254049]

4. Garon SL, Pavlos RK, White KD, Brown NJ, Stone CA Jr, Phillips EJ. Pharmacogenomics of offtarget adverse drug reactions.Br J Clin Pharmacol 2017; 83: 1896-911. [PubMed: 28345177]

5. McNeil BD, Pundir P, Meeker S, et al. Identification of a mast-cell-specific receptor crucial for pseudo-allergic drug reactions. Nature 2015; 519: 237-41. [PubMed: 25517090]

6. Oettgen HC. Chapter 23: immunobiology of IgE and IgE receptors In: Adkinson NF Jr, Bochner BS, Burks AW, et al. Middleton's allergy principles and practice, 8th edn Philadelphia, PA: Elsevier Saunders, 2014: 364-75.

7. White KD, Abe R, Ardern-Jones M, et al. SJS/TEN 2017: building multidisciplinary networks to drive science and translation.J Allergy Clin Immunol Pract 2018; 6: 38-69. [PubMed: 29310768]

8. Joint Task Force on Practice Parameter, American Academy of Allergy Asthma and Immunology and American College of Allergy Asthma and Immunology. Drug allergy: an updated practice parameter. Ann Allergy Asthma Immunol 2010; 105: 259-73. [PubMed: 20934625]

9. Dona I, Moreno E, Perez-Sanchez N, Andreu I,Hernandez Fernandez de Rojas D, Torres MJ. Update on quinolone allergy. Curr Allergy Rep 2017; 17: 56.

10. Mertes PM, Lambert M, Gueant-Rodriguez RM, et al. Perioperative anaphylaxis. Immunol Allergy Clin North Am 2009; 29: 429-51. [PubMed: 19563990]

11. Bigby M, Jick S, Jick H, Arndt K. Drug-induced cutaneous reactions. A report from the Boston Collaborative Drug Surveillance Program on 15,438 consecutive inpatients, 1975 to 1982. JAMA 1986; 256: 3358-63. [PubMed: 2946876]

12. Minhas JS, Wickner PG, Long AA, Banerji A, Blumenthal KG. Immune-mediated reactions to vancomycin: a systematic case review and analysis. Ann Allergy Asthma Immunol 2016; 116 : 544-53. [PubMed: 27156746]

13. Pavlos R, White KD, Wanjalla C, Mallal SA, Phillips EJ. Severe delayed drug reactions: role of genetics and viral infections. Immunol Allergy Clin North Am 2017; 37: 785-815. [PubMed: 28965641]

14. Chovel-Sella A, Ben Tov A, Lahav E, et al. Incidence of rash after amoxicillin treatment in children with infectious mononucleosis. Pediatrics 2013; 131: e1424-27. [PubMed: 23589810] 
15. Chung WH, Shih SR, Chang CF, et al. Clinicopathologic analysis of coxsackievirus a6 new variant induced widespread mucocutaneous bullous reactions mimicking severe cutaneous adverse reactions. J Infect Dis 2013; 208: 1968-78. [PubMed: 23904296]

16. Senst BL, Achusim LE, Genest RP, et al. Practical approach to determining costs and frequency of adverse drug events in a health care network. Am J Health Syst Pharm 2001; 58: 1126-32. [PubMed: 11449856]

17. Hug BL, Keohane C, Seger DL, Yoon C, Bates DW. The costs of adverse drug events in community hospitals. Jt Comm J Qual Patient Saf 2012; 38: 120-26. [PubMed: 22435229]

18. Miguel A, Azevedo LF, Araujo M, Pereira AC. Frequency of adverse drug reactions in hospitalized patients: a systematic review and meta-analysis. Pharmacoepidemiol Drug Saf 2012; 21: 1139-54. [PubMed: 22761169]

19. Blumenthal KG, Lai KH, Huang M, Wallace ZS, Wickner PG, Zhou L. Adverse and hypersensitivity reactions to prescription nonsteroidal anti-inflammatory agents in a large health care system. J Allergy Clin Immunol Pract 2017; 5: 737-43.e3. [PubMed: 28110055]

20. Sousa-Pinto B, Fonseca JA, Gomes ER. Frequency of self-reported drug allergy: a systematic review and meta-analysis with meta-regression. Ann Allergy Asthma Immunol 2017; 119: 36273.e2. [PubMed: 28779998]

21. Goss FR, Lai KH, Topaz M, et al. A value set for documenting adverse reactions in electronic health records. J Am Med Inform Assoc 2018; 25: 661-69. [PubMed: 29253169]

22. Thompson DF, Ramos CL. Antibiotic-induced rash in patients with infectious mononucleosis. Ann Pharmacother 2017; 51: 154-62. [PubMed: 27620494]

23. Hocqueloux L, Guinard J, Buret J, Causse X, Guigon A. Do penicillins really increase the frequency of a rash when given during Epstein-Barr virus primary infection? Clin Infect Dis 2013; 57: 1661-62. [PubMed: 23964089]

24. Macy E, Ho NJ. Multiple drug intolerance syndrome: prevalence, clinical characteristics, and management. Ann Allergy Asthma Immunol 2012; 108: 88-93. [PubMed: 22289726]

25. Zhou L, Dhopeshwarkar N, Blumenthal KG, et al. Drug allergies documented in electronic health records of a large healthcare system. Allergy 2016; 71: 1305-13. [PubMed: 26970431]

26. Gomes E, Cardoso MF, Praca F, Gomes L, Marino E, Demoly P. Self-reported drug allergy in a general adult Portuguese population. Clin Exp Allergy 2004; 34: 1597-601. [PubMed: 15479276]

27. Strom BL, Schinnar R, Apter AJ, et al. Absence of cross-reactivity between sulfonamide antibiotics and sulfonamide nonantibiotics. N Engl J Med 2003; 349: 1628-35. [PubMed: 14573734]

28. Mockenhaupt M, Viboud C, Dunant A, et al. Stevens-Johnson syndrome and toxic epidermal necrolysis: assessment of medication risks with emphasis on recently marketed drugs. The EuroSCAR-study. J Invest Dermatol 2008; 128: 35-44. [PubMed: 17805350]

29. Blumenthal KG, Wickner PG, Lau JJ, Zhou L. Stevens-Johnson syndrome and toxic epidermal necrolysis: a cross-sectional analysis of patients in an integrated allergy repository of a large health care system. J Allergy Clin Immunol Pract 2015; 3: 277-80.e1. [PubMed: 25609329]

30. Demoly P, Adkinson NF, Brockow K, et al. International Consensus on drug allergy. Allergy 2014; 69: 420-37. [PubMed: 24697291]

31. Kardaun SH, Sekula P, Valeyrie-Allanore L, et al. Drug reaction with eosinophilia and systemic symptoms (DRESS): an original multisystem adverse drug reaction. Results from the prospective RegiSCAR study. Br J Dermatol 2013; 169: 1071-80. [PubMed: 23855313]

32. Lin YF, Yang CH, Sindy H, et al. Severe cutaneous adverse reactions related to systemic antibiotics. Clin Infect Dis 2014; 58: 1377-85. [PubMed: 24599767]

33. Trubiano JA, Aung AK, Nguyen M, et al. A comparative analysis between antibiotic- and nonantibiotic-associated delayed cutaneous adverse drug reactions. J Allergy Clin Immunol Pract 2016; 4: 1187-93. [PubMed: 27283055]

34. Waldbott GL. Anaphylactic death from penicillin. JAMA 1949;139: 526-27.

35. van Dijk SM, Gardarsdottir H, Wassenberg MW, Oosterheert JJ, de Groot MC, Rockmann H. The high impact of penicillin allergy registration in hospitalized patients. J Allergy Clin Immunol Pract 2016; 4: 926-31. [PubMed: 27131826] 
36. Macy E, Poon KYT. Self-reported antibiotic allergy incidence and prevalence: age and sex effects. Am J Med 2009; 122: 778.e1-7.

37. Lee P, Shanson D. Results of a UK survey of fatal anaphylaxis after oral amoxicillin. J Antimicrob Chemother 2007; 60: 1172-73. [PubMed: 17761735]

38. Thornhill MH, Dayer MJ, Prendergast B, Baddour LM, Jones S, Lockhart PB. Incidence and nature of adverse reactions to antibiotics used as endocarditis prophylaxis. J Antimicrob Chemother 2015; 70: 2382-88. [PubMed: 25925595]

39. Dhopeshwarkar N, Sheikh A, Doan R, et al. Drug-induced anaphylaxis documented in electronic health records. J Allergy Clin Immunol Pract 2018; 10.1016/j.jaip.2018.06.010 (preprint).

40. Sogn DD. Prevention of allergic reactions to penicillin. J Allergy Clin Immunol 1986; 78: $1051-$ 52. [PubMed: 3782665]

41. Adkinson NF Jr. Risk factors for drug allergy. J Allergy Clin Immunol 1984; 74: 567-72. [PubMed: 6491103]

42. Chaby G, Valeyrie-Allanore L, Duong TA, et al. Severe cutaneous adverse reactions due to inappropriate medication use. Br J Dermatol 2018; 179: 329-36. [PubMed: 29352771]

43. Hsu DY, Brieva J, Silverberg NB, Silverberg JI. Morbidity and mortality of Stevens-Johnson syndrome and toxic epidermal necrolysis in United States adults. J Invest Dermatol 2016; 136: 1387-97. [PubMed: 27039263]

44. Macy E, Contreras R. Adverse reactions associated with oral and parenteral use of cephalosporins: a retrospective population-based analysis. J Allergy Clin Immunol 2015; 135: 745-52.e5. [PubMed: 25262461]

45. Barranco P, Lopez-Serrano MC. General and epidemiological aspects of allergic drug reactions. Clin Exper Allergy 1998; 28 (suppl 4): 61-62. [PubMed: 9761035]

46. Trubiano JA, Pai Mangalore R, Baey YW, et al. Old but not forgotten: antibiotic allergies in general medicine (the AGM Study). Med J Aust 2016; 204: 273. [PubMed: 27078602]

47. Lee CE, Zembower TR, Fotis MA, et al. The incidence of antimicrobial allergies in hospitalized patients: implications regarding prescribing patterns and emerging bacterial resistance. Arch Intern Med 2000; 160: 2819-22. [PubMed: 11025792]

48. MacFadden DR, LaDelfa A, Leen J, et al. Impact of reported $\beta$-lactam allergy on inpatient outcomes: a multicenter prospective cohort study. Clin Infect Dis 2016; 63: 904-10. [PubMed: 27402820]

49. Blumenthal KG, Li Y, Acker WW, et al. Multiple drug intolerance syndrome and multiple drug allergy syndrome: epidemiology and associations with anxiety and depression. Allergy 2018; 10.1111/all.13440 (preprint).

50. Huang KG, Cluzet V, Hamilton K, Fadugba O. The impact of reported $\beta$-lactam allergy in hospitalized patients with hematologic malignancies requiring antibiotics. Clin Infect Dis 2018; 67: 27-33. [PubMed: 29346543]

51. Trubiano JA, Leung VK, Chu MY, Worth LJ, Slavin MA, Thursky KA. The impact of antimicrobial allergy labels on antimicrobial usage in cancer patients. Antimicrob Resist Infect Control 2015; 4: 23. [PubMed: 26034582]

52. Coopman SA, Johnson RA, Platt R, Stern RS. Cutaneous disease and drug reactions in HIV infection. N Engl J Med 1993; 328: 1670-74. [PubMed: 8487826]

53. Lehloenya RJ, Dheda K. Cutaneous adverse drug reactions to anti-tuberculosis drugs: state of the art and into the future. Expert Rev Anti Infect Ther 2012; 10: 475-86. [PubMed: 22512756]

54. May SM, Motosue MS, Park MA. Dapsone is often tolerated in HIV-infected patients with history of sulfonamide antibiotic intolerance. J Allergy Clin Immunol Pract 2017; 5: 831-33. [PubMed: 28034550]

55. Petroni DH, Aitken ML, Ham E, et al. Approach to the evaluation of adverse antibiotic reactions in patients with cystic fibrosis. Ann Allergy Asthma Immunol 2016; 117: 378-81. [PubMed: 27590641]

56. Jenkins RE, Yaseen FS, Monshi MM, et al. $\beta$-Lactam antibiotics form distinct haptenic structures on albumin and activate drug-specific T-lymphocyte responses in multiallergic patients with cystic fibrosis. Chem Res Toxicol 2013; 26: 963-75. [PubMed: 23668298] 
57. Trubiano JA, Adkinson NF, Phillips EJ. Penicillin allergy is not necessarily forever. JAMA 2017; 318: 82-83. [PubMed: 28672303]

58. Romano A, Gaeta F, Valluzzi RL, Zaffiro A, Caruso C, Quaratino D. Natural evolution of skin-test sensitivity in patients with IgE-mediated hypersensitivity to cephalosporins. Allergy 2014; 69: 806-09. [PubMed: 24673580]

59. Blanca M, Romano A, Torres MJ, et al. Update on the evaluation of hypersensitivity reactions to betalactams. Allergy 2009; 64: 183-93. [PubMed: 19133923]

60. Bourke J, Pavlos R, James I, Phillips E. Improving the effectiveness of penicillin allergy delabeling. J Allergy Clin Immunol Pract 2015; 3: 365-74.e1. [PubMed: 25609352]

61. Mill C, Primeau MN, Medoff E, et al. Assessing the diagnostic properties of a graded oral provocation challenge for the diagnosis of immediate and nonimmediate reactions to amoxicillin in children. JAMA Pediatr 2016; 170: e160033. [PubMed: 27043788]

62. Mori F, Cianferoni A, Barni S, Pucci N, Rossi ME, Novembre E. Amoxicillin allergy in children: five-day drug provocation test in the diagnosis of nonimmediate reactions. J Allergy Clin Immunol Pract 2015; 3: 375-80.e1. [PubMed: 25609343]

63. Sacco KA, Bates A, Brigham TJ, Imam JS, Burton MC. Clinical outcomes following inpatient penicillin allergy testing: a systematic review and meta-analysis. Allergy 2017; 72: 1288-96. [PubMed: 28370003]

64. Macy E, Ngor EW. Safely diagnosing clinically significant penicillin allergy using only penicilloyl-poly-lysine, penicillin, and oral amoxicillin. J Allergy Clin Immunol Pract 2013; 1: 258-63. [PubMed: 24565482]

65. Tucker MH, Lomas CM, Ramchandar N, Waldram JD. Amoxicillin challenge without penicillin skin testing in evaluation of penicillin allergy in a cohort of Marine recruits. J Allergy Clin Immunol Pract 2017; 5: 813-15. [PubMed: 28341170]

66. Moreno E, Laffond E, Munoz-Bellido F, et al. Performance in real life of the European Network on Drug Allergy algorithm in immediate reactions to $\beta$-lactam antibiotics. Allergy 2016;71: 1787-90. [PubMed: 27543745]

67. Mota I, Gaspar A, Chambel M, Piedade S, Morais-Almeida M. Hypersensitivity to $\beta$-lactam antibiotics: a three-year study. Eur Ann Allergy Clin Immunol 2016; 48: 212-19. [PubMed: 27852424]

68. Vyles D, Adams J, Chiu A, Simpson P, Nimmer M, Brousseau DC. Allergy testing in children with low-risk penicillin allergy symptoms. Pediatrics 2017; 140: e20170471. [PubMed: 28674112]

69. Vyles D, Chiu A, Simpson P, Nimmer M, Adams J, Brousseau DC. Parent-reported penicillin allergy symptoms in the pediatric emergency department. Acad Pediatr 2017; 17: 251-55. [PubMed: 28274586]

70. Norton AE, Konvinse K, Phillips EJ, Broyles AD. Antibiotic allergy in pediatrics. Pediatrics 2018; 141: e20172497. [PubMed: 29700201]

71. Iammatteo M, Blumenthal KG, Saff R, Long AA, Banerji A. Safety and outcomes of test doses for the evaluation of adverse drug reactions: a 5-year retrospective review. J Allergy Clin Immunol Pract 2014; 2: 768-74. [PubMed: 25439369]

72. Kao L, Rajan J, Roy L, Kavosh E, Khan DA. Adverse reactions during drug challenges: a single US institution's experience. Ann Allergy Asthma Immunol 2013; 110: 86-91.e1. [PubMed: 23352526]

73. Messaad D, Sahla H, Benahmed S, Godard P, Bousquet J, Demoly P. Drug provocation tests in patients with a history suggesting an immediate drug hypersensitivity reaction. Ann Intern Med 2004; 140: 1001-06. [PubMed: 15197017]

74. Blumenthal KG, Shenoy ES, Huang M, et al. The impact of reporting a prior penicillin allergy on the treatment of methicillin-sensitive Staphylococcus aureus bacteremia. PLoS One 2016; 11: e0159406. [PubMed: 27438379]

75. Jeffres MN, Narayanan PP, Shuster JE, Schramm GE. Consequences of avoiding $\beta$-lactams in patients with $\beta$-lactam allergies. J Allergy Clin Immunol 2016; 137: 1148-53. [PubMed: 26688516]

76. US Centers for Disease Control and Prevention. Surveillance for surgical site infection (SSI) events. https://www.cdc.gov/nhsn/acute-care-hospital/ssi/index.html (accessed May 7, 2018). 
77. US Centers for Disease Control and Prevention. Antibiotic/antimicrobial resistance (AR/AMR): biggest threats and data. https://www.cdc.gov/drugresistance/biggest_threats.html (accessed May 7, 2018).

78. Macy E, Contreras R. Health care use and serious infection prevalence associated with penicillin "allergy" in hospitalized patients: a cohort study. J Allergy Clin Immunol 2014; 133: 790-96. [PubMed: 24188976]

79. Blumenthal K, Lu N, Zhang Y, Li Y, Walensky RP, Choi HK. Risk of methicillin-resistant Staphylococcus aureus and Clostridium difficile in patients with a documented penicillin allergy: a population-based matched cohort study. BMJ 2018; 361: k2400. [PubMed: 29950489]

80. Zimlichman E, Henderson D, Tamir O, et al. Health care-associated infections: a meta-analysis of costs and financial impact on the US health care system. JAMA Intern Med 2013; 173: 2039-46. [PubMed: 23999949]

81. Awad SS. Adherence to surgical care improvement project measures and post-operative surgical site infections. Surg Infect 2012; 13: 234-37.

82. Blumenthal KG, Ryan EE, Li Y, Lee H, Kuhlen JL, Shenoy ES. The impact of a reported penicillin allergy on surgical site infection risk. Clin Infect Dis 2018; 66: 329-36. [PubMed: 29361015]

83. Bratzler DW, Dellinger EP, Olsen KM, et al. Clinical practice guidelines for antimicrobial prophylaxis in surgery. Am J Health Syst Pharm 2013; 70: 195-283. [PubMed: 23327981]

84. Macy E, Blumenthal KG. Are cephalosporins safe for use in penicillin allergy without prior allergy evaluation? J Allergy Clin Immunol Pract 2018; 6: 82-89. [PubMed: 28958745]

85. Balch A, Wendelboe AM, Vesely SK, Bratzler DW. Antibiotic prophylaxis for surgical site infections as a risk factor for infection with Clostridium difficile. PLoS One 2017; 12: e0179117. [PubMed: 28622340]

86. Romanelli VA, Howie MB, Myerowitz PD, et al. Intraoperative and postoperative effects of vancomycin administration in cardiac surgery patients: a prospective, double-blind, randomized trial. Crit Care Med 1993; 21: 1124-31. [PubMed: 8339575]

87. Cook T, Harper N. Anaesthesia, surgery and life-threatening allergic reactions: report and findings of the royal college of anaesthetists' 6th national audit project. $2018 \mathrm{http}: / /$ www.nationalauditprojects.org.uk/NAP6Report (accessed Aug 25, 2018).

88. O'Neill J. Antimicrobial resistance: tackling a crisis for the health and wealth of nations. 2014 https://amr-review.org/sites/default/files/AMR\%20Review\%20Paper\%20-\%20Tackling\%20a $\% 20$ crisis $\% 20$ for $\% 20$ the $\% 20$ health $\% 20$ and $\% 20$ wealth\%20of\%20nations_1.pdf (accessed Nov 21, 2018).

89. Pollack LA, Srinivasan A. Core elements of hospital antibiotic stewardship programs from the Centers for Disease Control and Prevention. Clin Infect Dis 2014; 59 (suppl 3): S97-100. [PubMed: 25261548]

90. Ressner RA, Gada SM, Banks TA. Antimicrobial stewardship and the allergist: reclaiming our antibiotic armamentarium. Clin Infect Dis 2016; 62: 400-01. [PubMed: 26486707]

91. Barlam TF, Cosgrove SE, Abbo LM, et al. Implementing an antibiotic stewardship program: guidelines by the Infectious Diseases Society of America and the Society for Healthcare Epidemiology of America. Clin Infect Dis 2016; 62: e51-77. [PubMed: 27080992]

92. Bousquet PJ, Demoly P, Romano A, et al. Pharmacovigilance of drug allergy and hypersensitivity using the ENDA-DAHD database and the GALEN platform. The Galenda project. Allergy 2009; 64: 194-203. [PubMed: 19178398]

93. Blumenthal KG, Shenoy ES, Wolfson AR, et al. Addressing inpatient $\beta$-lactam allergies: a multihospital implementation. J Allergy Clin Immunol Pract 2017; 5: 616-25.e7. [PubMed: 28483315]

94. Krishna MT, Huissoon AP, Li M, et al. Enhancing antibiotic stewardship by tackling "spurious" penicillin allergy. Clin Exp Allerg 2017; 47: 1362-73.

95. Vaisman A, McCready J, Hicks S, Powis J. Optimizing preoperative prophylaxis in patients with reported $\beta$-lactam allergy: a novel extension of antimicrobial stewardship. J Antimicrob Chemother 2017; 72: 2657-60. [PubMed: 28605452]

96. Sogn DD, Evans R 3rd, Shepherd GM, et al. Results of the National Institute of Allergy and Infectious Diseases Collaborative Clinical Trial to test the predictive value of skin testing with 
major and minor penicillin derivatives in hospitalized adults. Arch Intern Med 1992; 152: 1025 32. [PubMed: 1580706]

97. AllerQuest, ALK-Abelló. PRE-PEN benzylpenicilloyl polylysine injection, solution [package insert]. 2013 https://www.penallergytest.com/wp-content/uploads/PRE-PEN-Package-Insert.pdf (accessed Aug 24, 2018).

98. Diater Terapéuticas. DAP-kit [package insert]. 2009 http://www.medecapharma.com/dap/dapamoxicillin-en.pdf (accessed Aug 24, 2018).

99. Torres MJ, Romano A, Celik G, et al. Approach to the diagnosis of drug hypersensitivity reactions: similarities and differences between Europe and North America. Clin Transl Allergy 2017; 7 : 7. [PubMed: 28293415]

100. Rimawi RH, Cook PP, Gooch M, et al. The impact of penicillin skin testing on clinical practice and antimicrobial stewardship. J Hosp Med 2013; 8: 341-45. [PubMed: 23553999]

101. Heil EL, Bork JT, Schmalzle SA, et al. Implementation of an infectious disease fellow-managed penicillin allergy skin testing service. Open Forum Infect Dis 2016; 3: ofw155. [PubMed: 27704011]

102. Chen JR, Khan DA. Evaluation of penicillin allergy in the hospitalized patient: opportunities for antimicrobial stewardship. Curr Allergy Asthma Rep 2017; 17: 40. [PubMed: 28540641]

103. Empedrad R, Darter AL, Earl HS, Gruchalla RS. Nonirritating intradermal skin test concentrations for commonly prescribed antibiotics. J Allergy Clin Immunol 2003; 112: 629-30. [PubMed: 13679828]

104. Vezir E, Dibek Misirlioglu E, Civelek E, et al. Direct oral provocation tests in non-immediate mild cutaneous reactions related to $\beta$-lactam antibiotics. Pediatr Allergy Immunol 2016; 27: 50 54. [PubMed: 26619970]

105. Petz LD. Immunologic cross-reactivity between penicillins and cephalosporins: a review. J Infect Dis 1978; 137 (suppl): S74-79. [PubMed: 77300]

106. Romano A, Gaeta F, Valluzzi RL, et al. IgE-mediated hypersensitivity to cephalosporins: crossreactivity and tolerability of alternative cephalosporins. J Allergy Clin Immunol 2015;136: 68591.e3. [PubMed: 25930196]

107. Romano A, Valluzzi RL, Caruso C, Maggioletti M, Quaratino D, Gaeta F. Cross-reactivity and tolerability of cephalosporins in patients with age-mediated hypersensitivity to penicillins. J Allergy Clin Immunol Pract 2018; 6: 1662-72. [PubMed: 29408440]

108. Solensky R, Earl HS, Gruchalla RS. Lack of penicillin resensitization in patients with a history of penicillin allergy after receiving repeated penicillin courses. Arch Intern Med 2002; 162: 822-26. [PubMed: 11926858]

109. Tonson la Tour A, Michelet M, Eigenmann PA, Caubet JC. Natural history of benign nonimmediate allergy to $\beta$-lactams in children: a prospective study in retreated patients after a positive and a negative provocation test. J Allergy Clin Immunol Pract 2018; 6: 1321-26. [PubMed: 29175371]

110. Confino-Cohen R, Rosman Y, Meir-Shafrir K, et al. Oral challenge without skin testing safely excludes clinically significant delayed-onset penicillin hypersensitivity. J Allergy Clin Immunol Pract 2017; 5: 669-75. [PubMed: 28483317]

111. Cernadas JR, Brockow K, Romano A, et al. General considerations on rapid desensitization for drug hypersensitivity - a consensus statement. Allergy 2010; 65: 1357-66. [PubMed: 20716314]

112. Legendre DP, Muzny CA, Marshall GD, Swiatlo E. Antibiotic hypersensitivity reactions and approaches to desensitization. Clin Infect Dis 2014; 58: 1140-48. [PubMed: 24368623]

113. Barbaud A, Collet E, Milpied B, et al. A multicentre study to determine the value and safety of drug patch tests for the three main classes of severe cutaneous adverse drug reactions. Br J Dermatol 2013; 168: 555-62. [PubMed: 23136927]

114. Brockow K, Garvey LH, Aberer W, et al. Skin test concentrations for systemically administered drugs-an ENDA/EAACI Drug Allergy Interest Group position paper. Allergy 2013; 68: 702-12. [PubMed: 23617635]

115. Romano A, Blanca M, Torres MJ, et al. Diagnosis of nonimmediate reactions to $\beta$-lactam antibiotics. Allergy 2004; 59: 1153-60. [PubMed: 15461594] 
116. Rive CM, Bourke J, Phillips EJ. Testing for drug hypersensitivity syndromes. Clin Biochem Rev 2013; 34: 15-38. [PubMed: 23592889]

117. Barbaud A. Drug patch testing in systemic cutaneous drug allergy.Toxicology 2005; 209: 209-16. [PubMed: 15767038]

118. Absar N, Daneshvar H, Beall G. Desensitization to trimethoprim/sulfamethoxazole in HIVinfected patients. J Allergy Clin Immunol 1994; 93: 1001-05. [PubMed: 8006304]

119. Beeler A, Engler O, Gerber BO, Pichler WJ. Long-lasting reactivity and high frequency of drugspecific T cells after severe systemic drug hypersensitivity reactions. J Allergy Clin Immunol 2006; 117: 455-62. [PubMed: 16461148]

120. National Institute for Health and Care Excellence. Using antimicrobial medicines safely and wisely to treat infections. 2015 https://www.nice.org.uk/guidance/ng15/resources/usingantimicrobial-medicines-safely-and-wisely-to-treat-infections-pdf-327009432517 (accessed Aug $25,2018)$.

121. US Centers for Disease Control and Prevention. Is it really a penicillin allergy? Evaluation and diagnosis of penicillin allergy for healthcare professionals. 2017 https://www.cdc.gov/antibioticuse/community/for-hcp/Penicillin-Allergy.html (accessed Nov 21, 2018).

122. Penicillin Allergy in Antibiotic Resistance Workgroup. Penicillin allergy testing should be performed routinely in patients with self-reported penicillin allergy. J Allergy Clin Immunol Pract 2017; 5: 333-34. [PubMed: 28283158]

123. Chen JR, Tarver SA, Alvarez KS, Tran T, Khan DA. A proactive approach to penicillin allergy testing in hospitalized patients. J Allergy Clin Immunol Pract 2017; 5: 686-93. [PubMed: 27888034]

124. Cook DJ, Barbara DW, Singh KE, Dearani JA. Penicillin skin testing in cardiac surgery. J Thorac Cardiovasc Surg 2014; 147: 1931-35. [PubMed: 24530197]

125. McDanel DL, Azar AE, Dowden AM, et al. Screening for $\beta$-lactam allergy in joint arthroplasty patients to improve surgical prophylaxis practice. J Arthroplasty 2017; 32: S101-08 [PubMed: 28236547]

126. Park M, Markus P, Matesic D, Li JT. Safety and effectiveness of a preoperative allergy clinic in decreasing vancomycin use in patients with a history of penicillin allergy. Ann Allergy Asthma Immunol 2006; 97: 681-87. [PubMed: 17165279]

127. Blumenthal KG, Wickner PG, Hurwitz S, et al. Tackling inpatient penicillin allergies: assessing tools for antimicrobial stewardship. J Allergy Clin Immunol 2017; 140: 154-61.e6. [PubMed: 28254470]

128. Antimicrobial hypersensitivity (revised 2014 Oct). In: eTG complete. Melbourne: Therapeutic Guidelines Ltd, 2015.

129. Joint Royal College of Physicians and Royal College of Pathologists Working Party. Allergy services: still not meeting the unmet need. $2010 \mathrm{https} / / / \mathrm{cdn}$.shopify.com/s/files/1/0924/4392/files/ allergy-services-still-not-meeting-the-unmet-need.pdf?1709961806511712341 (accessed May 9, 2018).

130. Dworzynski K, Ardern-Jones M, Nasser S, Guideline Development Group, National Institute for Health and Care Excellence. Diagnosis and management of drug allergy in adults, children and young people: summary of NICE guidance. BMJ 2014; 349: g4852. [PubMed: 25186447]

131. Trubiano JA, Beekmann SE, Worth LJ, et al. Improving antimicrobial stewardship by antibiotic allergy delabeling: evaluation of knowledge, attitude, and practices throughout the emerging infections network. Open Forum Infect Dis 2016; 3: ofw153 [PubMed: 27800527]

132. Trubiano JA, Worth LJ, Urbancic K, et al. Return to sender: the need to re-address patient antibiotic allergy labels in Australia and New Zealand. Intern Med J 2016; 46: 1311-17. [PubMed: 27527526]

133. Marwood J, Aguirrebarrena G, Kerr S, Welch SA, Rimmer J. De-labelling self-reported penicillin allergy within the emergency department through the use of skin tests and oral drug provocation testing. Emerg Med Australas 2017; 29: 509-15. [PubMed: 28378949]

134. Raja AS, Lindsell CJ, Bernstein JA, Codispoti CD, Moellman JJ. The use of penicillin skin testing to assess the prevalence of penicillin allergy in an emergency department setting. Ann Emerg Med 2009; 54: 72-77. [PubMed: 19217696] 
135. Bauer CS, Chiu AM, Zafra HT, et al. The value of an allergy and clinical immunology rotation at an academic tertiary medical center. Ann Allergy Asthma Immunol 2013; 110: 468-69.e9. [PubMed: 23706721]

136. Stukus DR, Green T, Montandon SV, Wada KJ. Deficits in allergy knowledge among physicians at academic medical centers. Ann Allergy Asthma Immunol 2015; 115: 51-55.e1. [PubMed: 26024806]

137. Staicu ML, Soni D, Conn KM, Ramsey A. A survey of inpatient practitioner knowledge of penicillin allergy at 2 community teaching hospitals. Ann Allergy Asthma Immunol 2017; 119: 42-47. [PubMed: 28539186]

138. Shah NS, Ridgway JP, Pettit N, Fahrenbach J, Robicsek A. Documenting penicillin allergy: the impact of inconsistency. PLoS One 2016; 11: e0150514. [PubMed: 26981866]

139. Khalil H, Leversha A, Khalil V. Drug allergy documentation-time for a change? Int J Clin Pharm 2011; 33: 610-13. [PubMed: 21614630]

140. Bouwmeester MC, Laberge N, Bussieres JF, Lebel D, Bailey B, Harel F. Program to remove incorrect allergy documentation in pediatrics medical records. Am J Health Syst Pharm 2001; 58: 1722-27. [PubMed: 11571814]

141. Kleris R, Lugar PL. Penicillin skin testing to evaluate penicillin allergy: outcome of testing results fails to affect clinical practice. J Allergy Clin Immunol 2017; 139: AB29.

142. Picard M, Paradis L, Nguyen M, Begin P, Paradis J, Des Roches A. Outpatient penicillin use after negative skin testing and drug challenge in a pediatric population. Allergy Asthma Proc 2012;33: 160-64. [PubMed: 22450448] 


\section{Search Strategy and Selection Criteria}

We searched MEDLINE from January 1, 2005 through April 30, 2018, but did not exclude commonly referenced landmark articles published prior to 2005. Primary search terms included: "drug" "antibiotics" "drug-induced" "penicillin" "beta-lactam" "sulfonamide" "nevirapine" "abacavir" "antiretroviral” "rifampin" "rifamycin" "vancomycin" "fluoroquinolone" "anesthesia" "itch" "erythema" "pruritus" "rhinitis" "wheezing" "urticaria" "hive" "angioedema" "edema" "swelling" "anaphylaxis" "serum sickness" "fever" "rash" "eczema" "contact" "dermatitis" "maculopapular" "interstitial” "nephritis" "erythema multiforme" "exfoliative dermatitis" "drug reaction with eosinophilia and systemic symptoms" "drug-induced hypersensitivity syndrome" "severe cutaneous adverse reaction" "Stevens-Johnson syndrome" "toxic epidermal necrolysis" "liver" "DILI" "hepatotoxicity" "acute generalized exanthematous pustulosis" "fixed drug reaction" "linear IgA exanthem" "allergy" "hypersensitivity" "cross-reactivity." Priority review was given to studies with more rigorous study designs, those with more numbers or diversity of patients, and those published in higher-quality journals. We excluded clinical drug trials, case reports, and animal model studies. Additional articles were identified through review of all reference lists from relevant articles identified by this search strategy. Review articles, practice parameters, and position statements were included to provide readers with additional resources on this topic. Detailed sources on primary severe cutaneous adverse reaction (SCAR) data were excluded given a recent Seminar. ${ }^{[1]}$ 


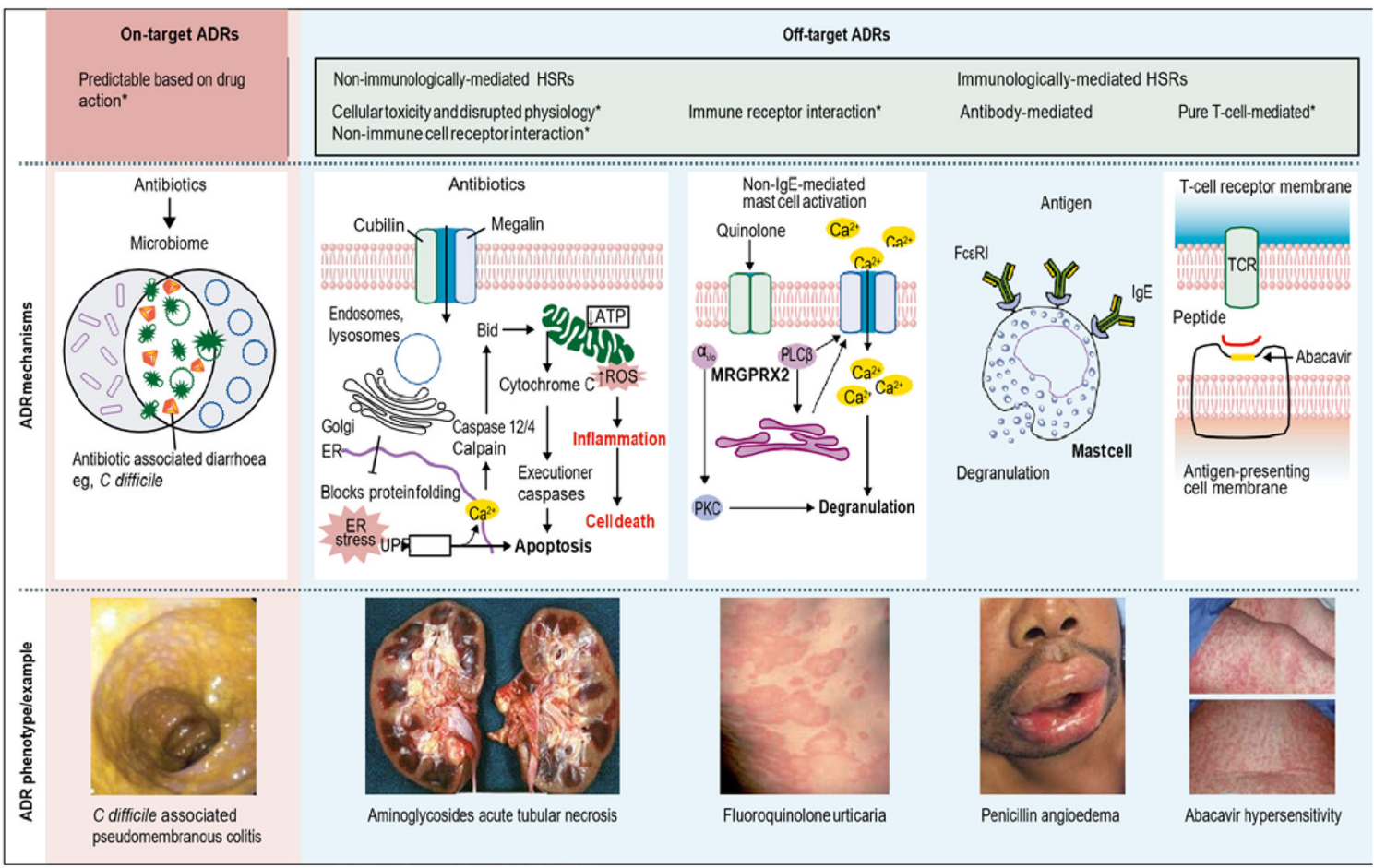

Figure 1:

Classification of on-target and off-target ADRS Pink panel illustrates an example of an ontarget ADR. Blue panel (left) illustrates non-immunologically-mediated off-target effects: direct cellular toxicity or disruption of normal physiology, interaction with non-immune receptors, and interaction with immune receptors (eg, non-IgE-mediated mast-cell activation via G-protein coupled receptors). Blue panel (right) shows immunologically mediated adaptive immune responses (antibody-mediated [eg, $\mathrm{IgE}]$ immediate reactions or T-cellmediated delayed reactions). Predisposition to both on-target and off-target reactions is driven by genetic variation, but also ecological factors that can vary over the course of an individual's lifetime. ADR=adverse drug reaction. Bid=BH3 interacting-domain death. $\mathrm{C}$ difficile $=$ Clostridioides difficile. $\mathrm{ER}=$ endoplasmic reticulum. FceR1=high-affinity $\operatorname{IgE}$ receptor. $\mathrm{HSR}=$ hypersensitivity reaction. $\mathrm{MRGPRX} 2=\mathrm{MAS}$-related G-protein coupled receptor member $\mathrm{X} 2$. PKC=protein kinase $\mathrm{C}$. PLC $\beta=$ phospholipase $\mathrm{C} \beta$. ROS=reactive oxygen species. $\mathrm{TCR}=\mathrm{T}$ cell receptor. $\mathrm{UPR}=$ unfolded protein response. $*$ Dose-dependent. Reproduced from Peter et al. ${ }^{[2]}$ 


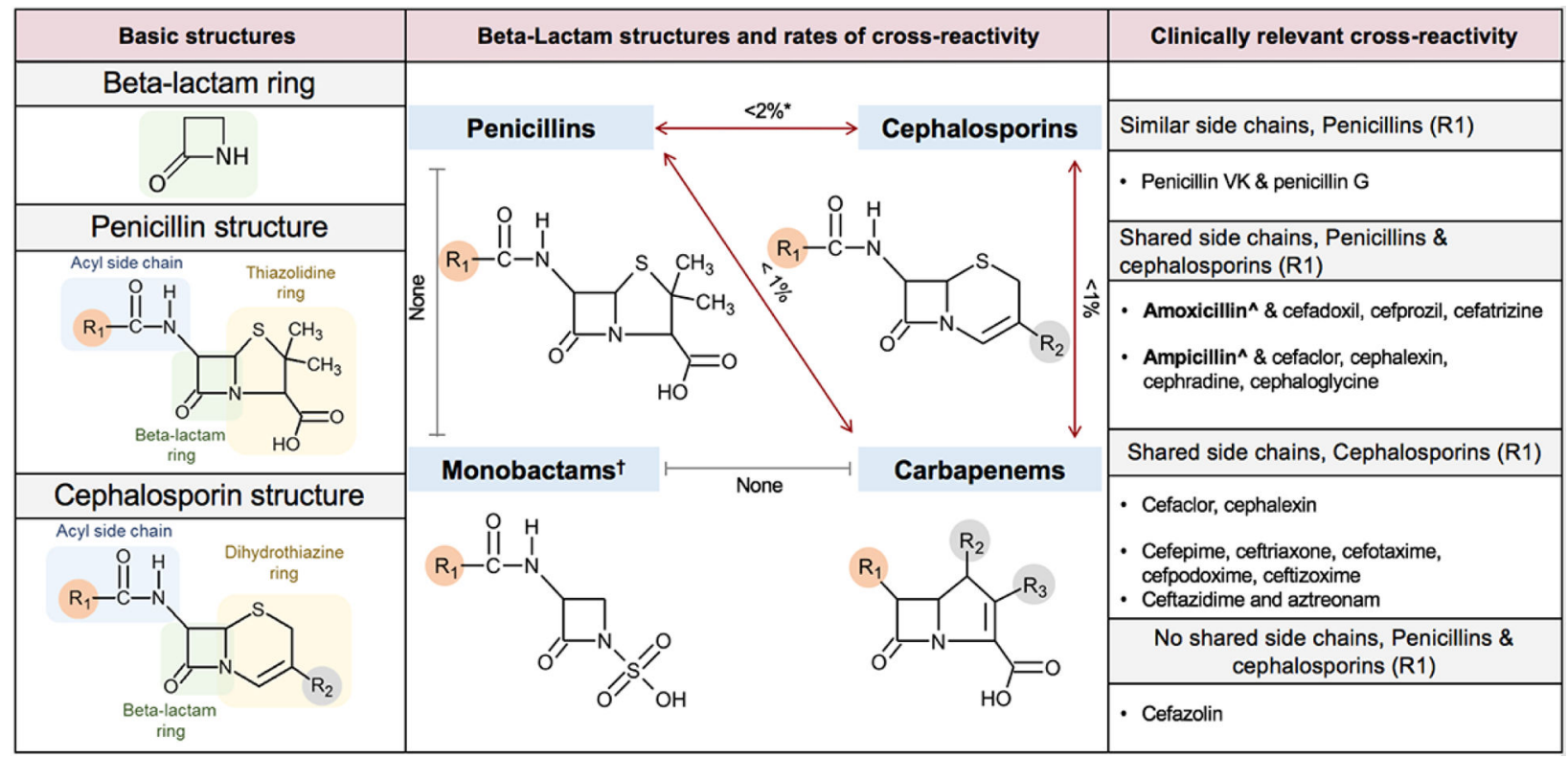

Figure 2:

$\beta$-Lactam structure and cross-reactivity $\beta$-Lactam antibiotics include penicillins, cephalosporins, carbapenems, and monobactams. Cross-reactivity is possible through the core $\beta$-lactam ring, adjacent thiazolidine (penicillin) or dihydrothiazine (cephalosporin) ring, and also from a side chain, R1, or R2 group (left panel). Cephalosporins have both an R1 and R2 group and penicillins only an R1. Despite varied mechanisms, true cross-reactivity is largely based on R1 side chains. Identical side chains in patients with IgE-mediated allergy pose the highest risk. However, cross-reactivity from side chains that are similar, but not identical, and from R2 group similarity is possible and reported. The centre panel demonstrates the structure and rates of cross-reactivity between penicillins, cephalosporins, carbapenems, and monobactams. The right panel details the most clinically important crossreactivity considerations. *Except for shared group aminopenicillins and cephalosporins. $\dagger$ Monobactams have no shared cross-reactivity with other $\beta$-lactams, with the exception for aztreonam and ceftazidime, which share an identical R1. $\$$ Amoxicillin and ampicillin are structurally similar aminopenicillins and should be considered clinically cross-reactive with each other and the respective cephalosporins with shared R1 groups listed in the figure.

Similar considerations exist for the aminocephalosporins. 


High $\begin{aligned} & \text { Mucosalinvolvement(eg, SJS)orprofoundskindesquamation } \\ & \text { Organ (eg, kidney) involvement or vital sign change } \\ & \text { Emergency visit or hospital admission } \\ & \text { Epinephrine use } \\ & \text { Rash with high or protracted fever } \\ & \text { Parenteral steroid use } \\ & \begin{array}{l}\text { Skin biopsy performed } \\ \text { Oral steroid use } \\ \text { Cutaneous symptoms only } \\ \text { Antihistamines used } \\ \text { Remote reactions (>10 years ago) } \\ \text { No therapy was needed }\end{array}\end{aligned}$

Figure 3:

Patient-reported history for risk stratification When limited allergy details are available, patient-reported historical details can be used to distinguish patients at high and low risk. In the case of penicillin allergy, patients with low risk histories are unlikely to be allergic and could be referred on large scales for allergy evaluations. When details are available about the purported reaction, the following questions are important components of the drug allergy history. (1) What were the symptoms? (raised, red, itchy spots with each lesion lasting less than $24 \mathrm{~h}$ [hives or urticaria]; swelling of the mouth, eyes, lips, or tongue [angioedema]; blisters or ulcers involving the lips, mouth, eyes, urethra, vagina, or peeling skin [severe type IV HSRs, SCARs]; respiratory or haemodynamic changes [anaphylaxis]; joint pains [serum sickness and serum-sickness like reaction]; organs involvement such as kidneys, lungs, or liver [severe type IV HSRs]). (2) What was the timing of the reaction after taking penicillin [minutes, hours, or days later]? Was it after the first dose or after multiple doses? (3) How long ago did the reaction happen? (4) How was the reaction treated? Was there a need for urgent care or was epinephrine administered? (5) Has the patient tolerated similar medications, such as ampicillin, amoxicillin, or cephalexin since the penicillin reaction? 


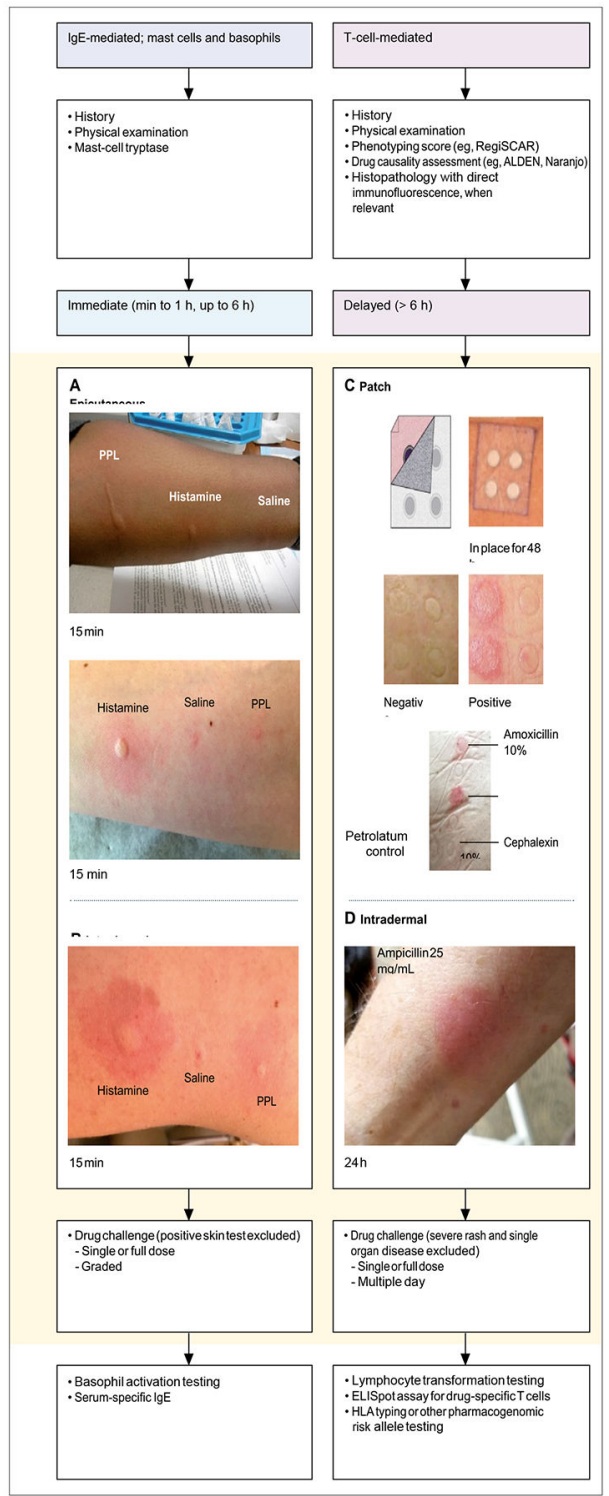

Figure 4:

Diagnostic approach to antibiotic allergy Immediate reactions commonly occur within $1 \mathrm{~h}$ but can occur up to $6 \mathrm{~h}$ after drug administration. Serum tryptase drawn 30-90 min after reaction onset is a useful biomarker to help differentiate anaphylaxis from non-IgE-mediated mast-cell activation. Drug-specific diagnostic tests for immediate reactions include (A) epicutaneous skin testing (ie, prick, puncture, or scratch) and (B) intradermal skin testing. The definition of a positive penicillin skin test varies globally. ${ }^{[96-98]}$ Delayed reactions typically occur in more than $6 \mathrm{~h}$ and up to 8 weeks after drug exposure and can occur after drug discontinuation. Testing for delayed reactions varies geographically and is not standardised. In-vivo testing for delayed reactions can include (C) patch testing, in which non-irritant drug concentrations in a base vehicle are applied by a Finn chamber and adhesive tape for $48 \mathrm{~h}$ and are read at $96 \mathrm{~h}$ and 1 week, or (D) delayed intradermal testing, in which results are read $24 \mathrm{~h}$ and $48 \mathrm{~h}$ after the drug solution is injected. Drug challenge, when 
safe to perform, is often the final step to confirm or exclude a drug allergy, after negative epicutaneous, immediate or delayed intradermal, or patch testing. In immediate reactions, drug challenges can feature a single full dose or be graded, with 2 to 3 dosing increments. In delayed reactions, dosing can be continued for multiple days but might be considered to be an unnecessary exposure to antibiotics. Drug challenge is contraindicated for SCAR and single-organ disease. Several additional ex-vivo and in-vitro diagnostic options are available in some subspecialty centres but are currently at the level of research tools that require further validation. See appendix. ALDEN=an algorithm for assessment of drug causality in Stevens-Johnson syndrome and toxic epidermal necrolysis. ELISpot=enzyme-linked immunosorbent assay. Naranjo=an adverse drug reaction probability scale that can be used to assess causality for any adverse drug reaction.

PPL=penicilloyl-polylysine. RegiSCAR=the European Registry of Severe Cutaneous Adverse Reactions to Drugs and Collection of Biological Samples group.

SCAR=severe cutaneous adverse reaction. 


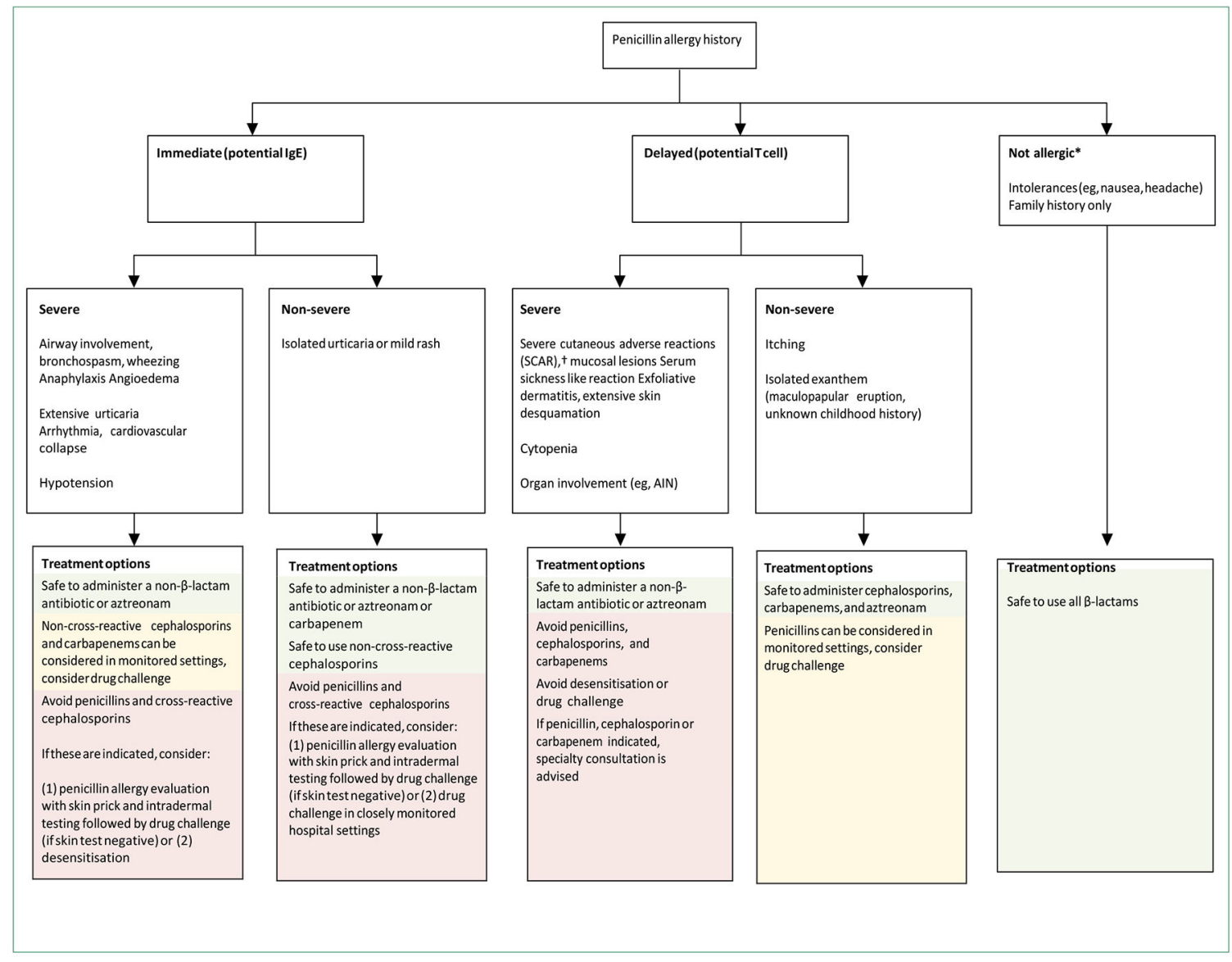

Figure 5:

Treatment algorithm for patients with penicillin allergy histories This algorithm, adapted from expert opinion, published studies, and guidelines, ${ }^{[93,127,128]}$ can be used to identify how to optimally prescribe $\beta$-lactam antibiotics acutely to patients with prior penicillin allergies. Reactions are divided into those with immediate and delayed onset, with reactions subsequently grouped as severe and non-severe. $\mathrm{ADR}=$ adverse drug reaction. $\mathrm{AGEP}=$ acute generalised exanthematous pustulosis. AIN=acute interstitial nephritis. DRESS=drug reaction with eosinophilia and systemic symptoms. SJS/TEN=Stevens-Johnson syndrome and toxic epidermal necrolysis.

*Non-immune mediated ADRs are typically pharmacologically predictable side effects which do not preclude penicillin usage. †SCARS include DRESS, SJS/TEN, and AGEP (table 2). 


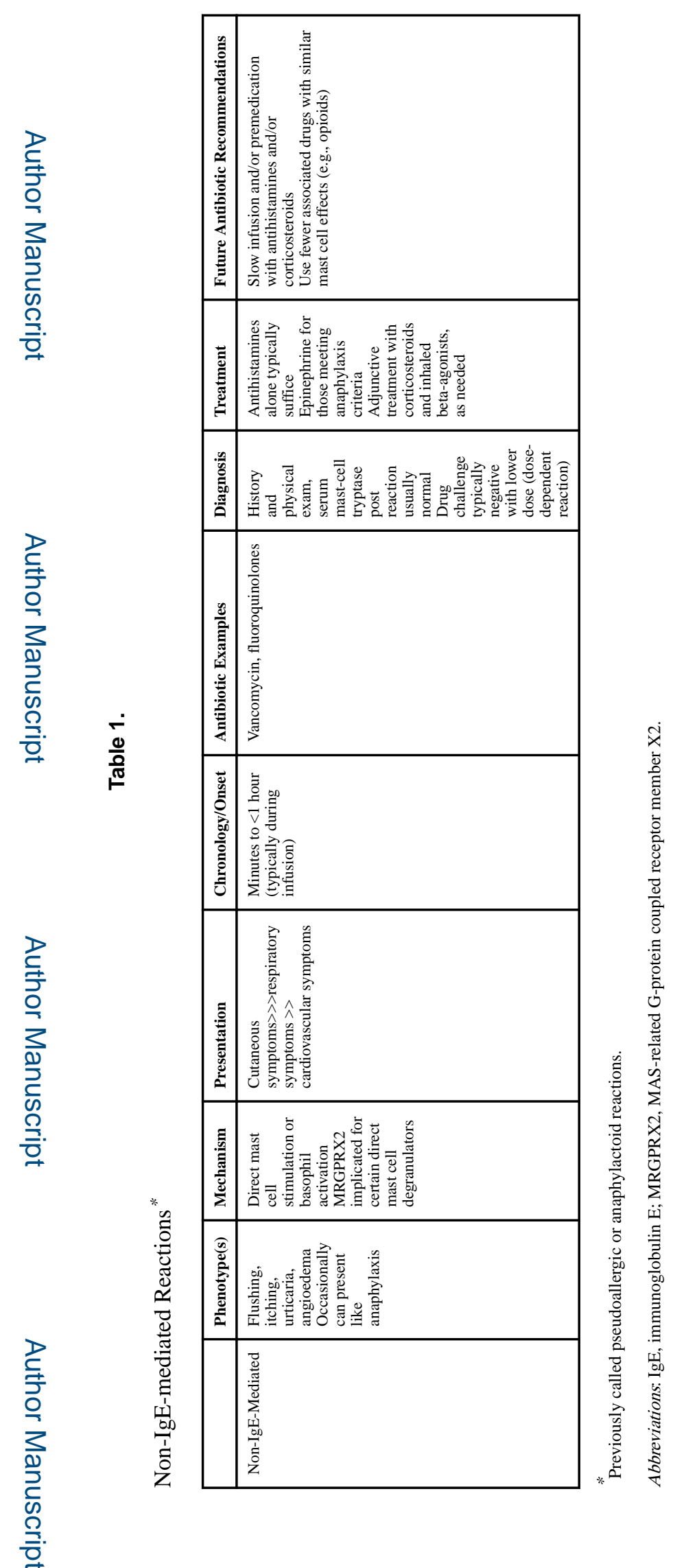

Lancet. Author manuscript; available in PMC 2019 June 13. 


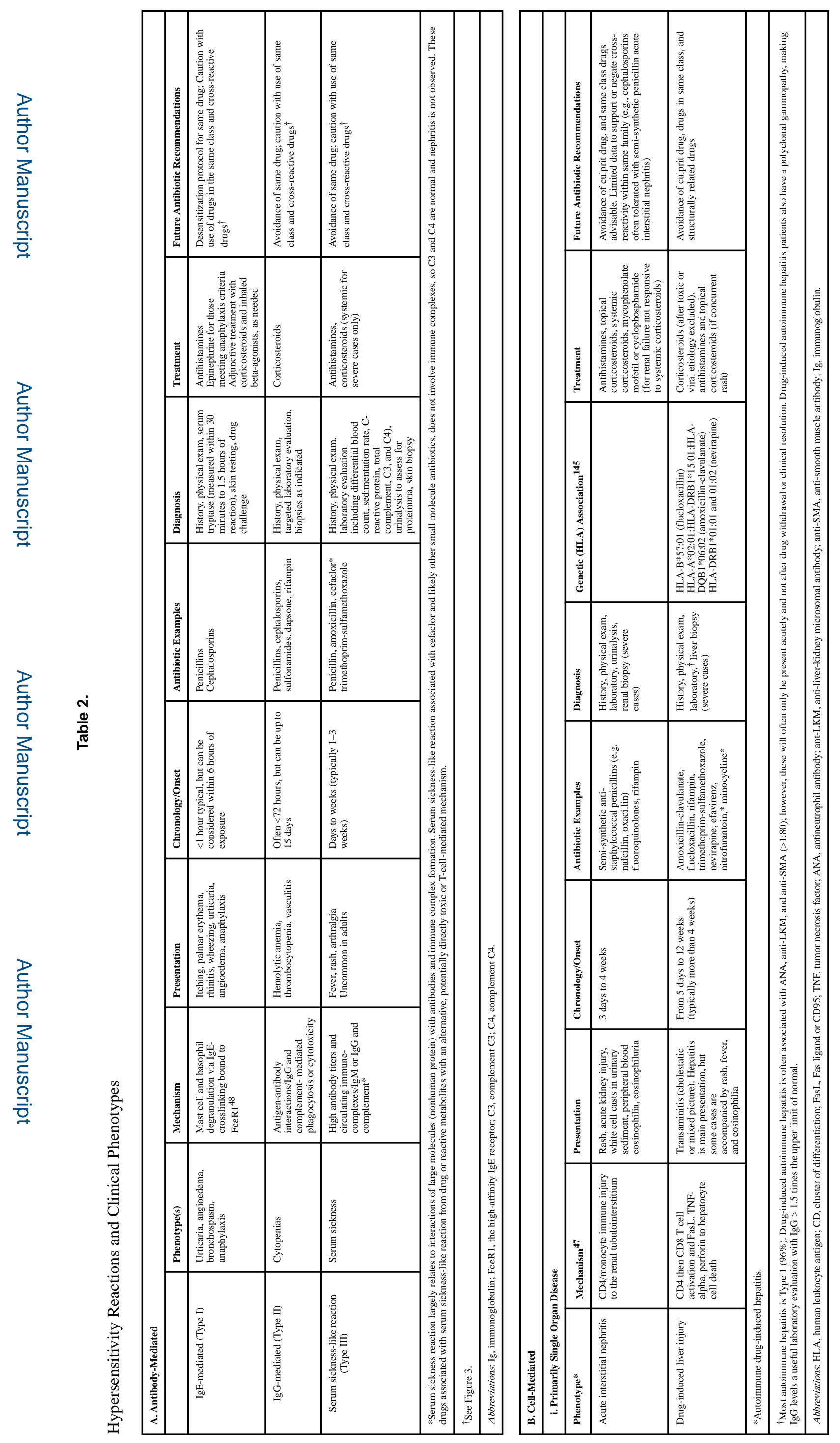




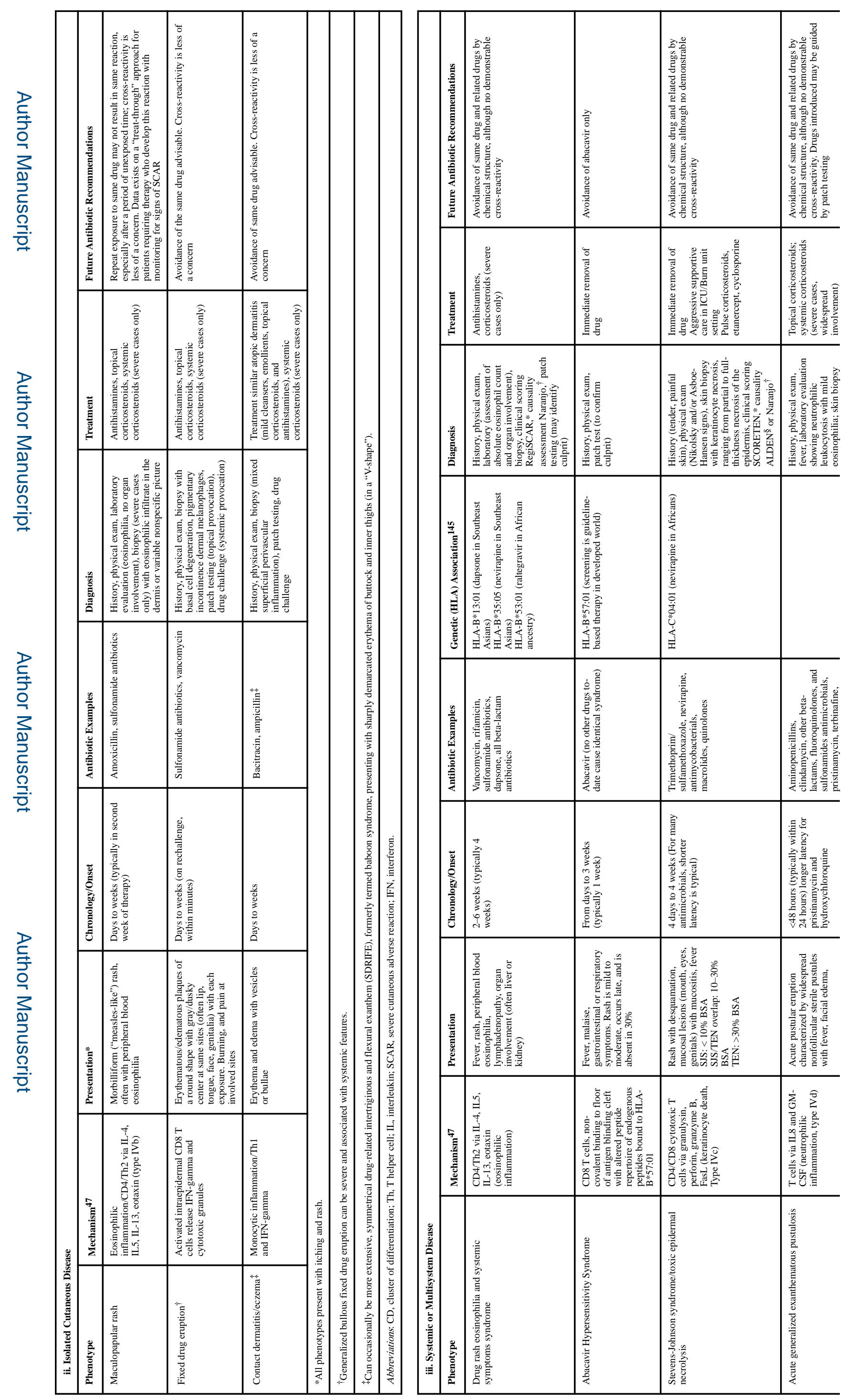




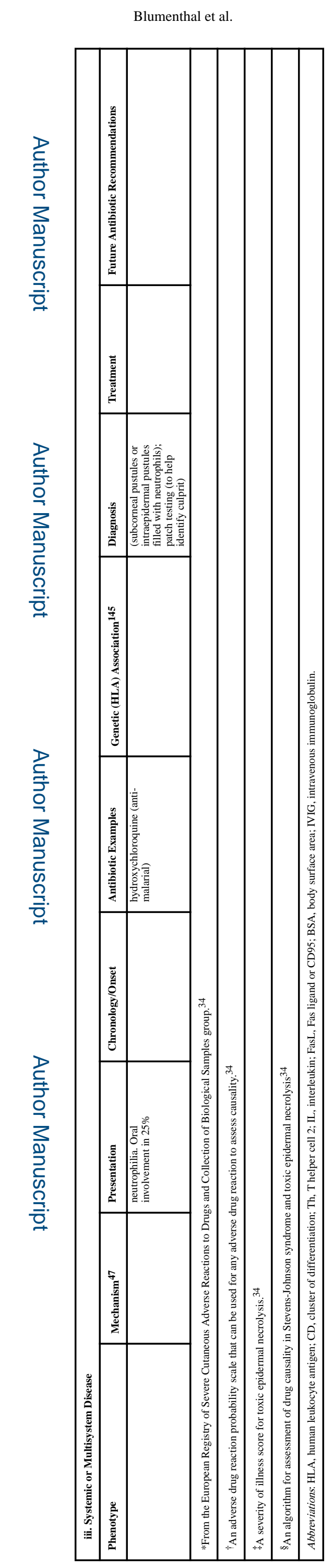

Lancet. Author manuscript; available in PMC 2019 June 13. 